\title{
ARTÍCULO
}

\section{LA DEMANDA Y OFERTA DE EDUCACIÓN TEMPRANA EN CHILE*}

\author{
Loreto Reyes \\ Ministerio de Hacienda \\ Sergio Urzúa \\ University of Maryland $y$ \\ Centro de Estudios Públicos
}

Resumen: Éste es el primer estudio en analizar la demanda y oferta de educación parvularia en América Latina, específicamente en Chile. La importancia de este tema se explica por

LORETO Reyes. Ingeniero Comercial, Universidad de Chile, y Magíster en Economía de la misma universidad. Analista del Ministerio de Hacienda, Santiago, Chile. Dirección electrónica: lreyes@hacienda.gov.cl.

Sergio Urzúa. Economista jefe del área de políticas sociales del Centro de Estudios Públicos. Profesor de la Universidad de Maryland y de la Universidad de Chile (surzua@cepchile.cl).

* Agradecemos los comentarios de dos anónimos árbitros de Estudios Públicos, de la Fundación Integra y del Departamento de Estudios en Primera Infancia del Ministerio de Educación, a quienes fue presentado este trabajo. Agradecemos la colaboración del Ministerio del Trabajo y de la Fundación Integra por la información entregada para desarrollar este estudio. La utilización de la información relativa a la Ficha de Protección Social (FPS) fue posible por el convenio suscrito por el Ministerio de Desarrollo Social y Sergio Urzúa. El análisis de estos datos fue realizado en el contexto del diseño del programa Ingreso Ético Familiar. Los datos fueron manejados reguardando todos los protocolos de seguridad descritos en el convenio. El documento no presenta información que permita identificar a personas o familias. Sergio Urzúa agradece el apoyo del Centro de Microdatos de la Universidad de Chile a través de la iniciativa Milenio del Ministerio de Economía de Chile, Proyecto NS10004.

Estudios Públicos, 126 (otoño 2012). 
el reciente aumento en la cobertura preescolar en la región, la necesidad de entender los determinantes de las decisiones de los padres de enviar a sus hijos a los centros (demanda), la caracterización de los centros que entregan los servicios de educación temprana (oferta) y los desafíos del diseño de políticas públicas en esta área. Nuestro análisis utiliza diversas fuentes de información. Respecto de la oferta, presentamos evidencia que sugiere problemas en la localización geográfica de los centros y en la coordinación entre los proveedores públicos. Respecto de la demanda, nuestros resultados indican que la distancia al centro, la educación de la madre y la situación de empleo son elementos importantes en la elección del establecimiento al que asisten los menores.

Palabras clave: primera infancia, educación temprana, capacidad y matrícula.

Recibido: mayo 2012; aceptado: mayo 2012.

\section{DEMAND AND SUPPLY OF EARLY EDUCATION IN CHILE}

Abstract: This is the first article analyzing the demand and supply of preschool education in Latin America, specifically in Chile. The importance of this topic is explained by the recent expansion of childcare public provision in Chile, the needs for understanding why/when/how parents decide whether to enroll their children (demand side), the role of the providers (supply side), and the challenges associated with the design of effective public policies in this critical area. The empirical analysis is carried out using several sources of information. We present evidence suggesting the existence of problems in the geographic distribution of childcare centers as well as the needs of better coordination between the two public providers (supply side). Our results also show that the distance to preschool centers, mother's education and the employment situation among the family members are important elements determining parents' enrollment decision.

Keywords: early chilhood, early education, capacity and enrollment.

Received: May 2012; accepted: May 2012. 


\section{Introducción}

$\mathrm{H}$ ros años de vida en el desarrollo del ser humano. Distintas investigaciones en el área de la neurociencia, han demostrado que los primeros cinco años de vida constituyen una etapa crítica para nuestro desarrollo (Knudsen et al., 2006). Durante este período se desarrollan gran parte de las funciones y estructuras cerebrales básicas determinantes de las habilidades y competencias futuras (Shonkoff and Phillips, 2000). Debido a que gran parte de las desigualdades sociales se originan en el ambiente que rodea la primera infancia, se argumenta que las intervenciones tempranas serían más productivas y menos costosas para remediar los efectos negativos de ambientes iniciales adversos que aquellas realizadas a edades posteriores (Carneiro y Heckman, 2003; Heckman, 2006). Diversos programas experimentales avalan la efectividad de estas intervenciones sobre el desarrollo cognitivo, desempeño educativo e incluso sobre otros indicadores sociales como la probabilidad de no participar en el mercado laboral y cometer actos ilícitos (consumo de drogas y delincuencia) (Barnet, 1995; Cuhna et al., 2005; Schady, 2005).

No obstante lo anterior, la literatura respecto de la forma en que se organiza la demanda y la oferta por educación preescolar es escasa. Estudios a nivel internacional analizan el impacto de las decisiones de matrícula sobre el desempeño cognitivo de los menores (Bernal y Keane 2010, 2011). Asimismo, existe escasa evidencia sobre los factores subyacentes a la forma en que compiten los proveedores públicos y privados en este mercado y si las decisiones de localización responden a un ordenamiento estratégico para capturar demanda. Kitano y Uda (2007) analizan el grado de accesibilidad de los jardines infantiles en dos provincias de Japón utilizando información georeferenciada de los hogares de los menores y de los centros. Por otra parte, Herbst y Tekin (2010) estudian el impacto de la ubicación de las agencias de servicios sociales sobre la probabilidad de obtener un subsidio de cuidado infantil. En este estudio desarrollamos un enfoque en esta línea.

En América Latina no existe evidencia sobre el comportamiento de la demanda y la oferta de educación temprana. Para Chile, los estudios existentes abordan la educación preescolar desde una perspectiva cualitativa (descriptiva) utilizando datos agregados de caracterización socioeconómica, con el fin de hacer recomendaciones respecto a la institucionalidad del sector y los mecanismos de financiamiento del sis- 
tema (Mineduc 2001, 2009; Tokman, 2010) ${ }^{1}$. Una excepción es NoboaHidalgo y Urzúa (2012) que combinan información de la disponibilidad local de salas cuna en 36 comunas de Chile para estudiar la demanda de las familias por sus servicios.

Sorprende el poco conocimiento generado en torno a este tema, en especial si consideramos los significativos aumentos en la cobertura preescolar observados a nivel mundial y la evidencia en torno a la importancia de una educación temprana de calidad (Engle et al., 2007; Heckman, 2006; Behrman y Urzúa, 2012).

Chile no es ajeno a ambos fenómenos. Durante los últimos años ha habido una significativa expansión de los programas de protección social orientados a la protección integral de la primera infancia. En particular, durante el período 2006-2010 se crearon más de 113.000 nuevos cupos en salas cuna y jardines infantiles, lo que significó un aumento de casi $500 \%$ en la oferta pública de salas cuna (niños menores de 2 años) y de un 55\% en la oferta pública de jardines infantiles en el nivel medio (es decir, niños mayores de 2 y menores de 4 años) ${ }^{2}$. Sin embargo, este explosivo aumento no estuvo acompañando de un plan estratégico acorde a las características y necesidades de la demanda y oferta. Como resultado, aspectos fundamentales ligados a la calidad de los servicios ofrecidos y a la localización geográfica de los centros, no fueron considerados como parte del diseño y hasta hoy permanecen como necesidades latentes en el sector.

En este estudio, analizamos el mercado de los centros de enseñanza preescolar en Chile (particularmente para la Región Metropoli-

1 Los estudios existentes analizan los determinantes de elección de establecimientos en el sistema escolar (educación básica y media) no así en el sector preescolar (por ejemplo Elacqua et al., 2006; Gallego y Hernando, 2008; Sapelli y Torche, 2002). En particular, Chumacero et al. (2008) y Gallego y Hernando (2009) utilizan distintas medidas de distancias a las escuelas para analizar los factores que explican las decisiones de los padres respecto de los establecimientos a los que asisten sus hijos.

2 Dentro de las metas planteadas en el programa Chile Crece Contigo (ChCC) para el período 2006-2010 estaba la creación de 70.000 nuevos cupos en salas cuna y 43.000 en jardines infantiles Junji e Integra. Como resultado, en este período el número de cupos en salas cuna Junji e Integra (orientadas a la atención de niños menores a 2 años) a nivel nacional se incrementó desde 14.402 a 85.000 mientras que los cupos en jardines infantiles (para niños mayores a 2 años y menores a 4 años) de las mismas instituciones aumentó desde 82.400 a 127.472 (Mideplan, 2010). 
tana), utilizando datos de niños entre 0 y 3 años $^{3}$ inscritos en la Ficha de Protección Social (FPS) y registros de matrículas al año 2010 de las dos instituciones públicas encargadas de proveer servicios de cuidado infantil: la Junta Nacional de Jardines Infantiles (Junji) y la Fundación Integra. Éste constituye el primer esfuerzo por caracterizar la población infantil chilena que asiste a salas cuna y jardines infantiles, a la que no asiste (pero potencialmente podría hacerlo) y a los proveedores de enseñanza preescolar.

Nuestra perspectiva empírica emplea información georeferenciada de los menores que asisten, de quienes potencialmente podrían asistir a centros de educación preescolar, y de jardines infantiles y salas cuna administrados por Junji e Integra. Con esta información analizamos el grado de consonancia entre la localización de los centros de educación parvularia y la ubicación de los menores que asisten a estos establecimientos. De esta forma, utilizamos la información disponible para evaluar:

i) si la ubicación de los centros responde a la existencia de una demanda efectiva y latente cercana,

ii) si la ubicación de los centros de ambas instituciones se explica por un ordenamiento estratégico de la demanda y de la oferta y,

iii) si la inasistencia a la educación parvularia está influenciada por la disponibilidad de cupos en establecimientos cercanos al hogar.

Nuestro estudio además extiende el análisis a otros dos importantes aspectos. Primero, examinamos el marco institucional vigente en el sector. En particular, identificamos a los principales proveedores y evaluamos el grado de coordinación entre éstos respecto de las decisiones de ubicación de los centros y sus características ${ }^{4}$. Segundo, analizamos modelos de elección (matrícula) utilizando la información a niveles de hogares y centros. Esto nos permite además evaluar la importancia en la decisión de enviar un menor a un establecimiento preescolar en función de la educación de los padres, la situación de vulnerabilidad del hogar, como también la situación de empleo de los padres.

${ }^{3}$ Este grupo es especialmente importante considerando que gran parte de la expansión de la oferta efectuada entre 2006 y 2010 estuvo orientada a este segmento.

${ }^{4}$ Otorgamos especial énfasis al estudio de la institucionalidad del sector debido a que determina la forma en que las distintas instituciones coordinan sus decisiones de localización y provisión de servicios (niveles educacionales a los que atienden, cupos ofrecidos, entre otros). Como veremos más adelante, estos dos últimos aspectos serán relevantes para analizar el equilibro entre la oferta y demanda por servicios preescolares (véase sección 5). 
En definitiva, en este trabajo se analiza la educación temprana en Chile utilizando fuentes inéditas de información, incorporando a su vez el análisis de ubicaciones geográficas al estudio de la demanda y oferta. Nuestros resultados indican que la distancia hogar-jardín es un elemento importante en la decisión de envío y en la elección final del centro al que asisten los menores. En promedio, los niños entre 0 y 3 años matriculados en Junji e Integra se ubican a 2 kilómetros del centro al que asisten, mientras que la distancia promedio desde el hogar al centro más cercano es de 720 metros. En el caso de los niños que no están matriculados, la distancia promedio hogar-centro más cercano es de 820 metros. Adicionalmente, estimamos que la probabilidad de asistir a un centro preescolar Junji o Integra se ve afectada positivamente por la edad de los menores (un año adicional incrementa la probabilidad en 6,2 puntos porcentuales) y la condición de empleabilidad de las madres (los hijos de madres ocupadas presentan 2,3 puntos porcentuales más de probabilidad de asistir a centros públicos en relación a los hijos de madres inactivas). Por el contrario, la probabilidad de asistencia a Junji o Integra disminuye con la educación de la madre (en promedio, los hijos de madres con más de doce años de escolaridad tienen aproximadamente 2 puntos porcentuales menos de probabilidad de asistir a establecimientos públicos), vulnerabilidad del hogar (medida a través del quintil en FPS) y la distancia desde el hogar al centro más cercano (niños cuyo centro más cercano está ubicado a más de cuatro kilómetros del hogar, tienen 26 puntos porcentuales menos de probabilidad de asistir a Junji o Integra).

Nuestros resultados también indican la existencia de problemas en la distribución de la oferta. Existen sectores con baja presencia de establecimientos, especialmente en los primeros niveles (sala cuna menor y sala cuna mayor) $)^{5}$. Respecto del grado de concentración de los centros Junji e Integra, nuestros resultados señalan que sobre el 50\% de los centros Junji tienen al menos un centro Integra situado a menos de 1 kilómetro de distancia y que la distancia promedio entre ambos tipos de centros es 550 metros. Asimismo, nuestro análisis identifica centros en los que la matrícula declarada excede la capacidad total de cupos ofre-

${ }^{5}$ Entre los 0 y 3 años, los niños pueden asistir a Sala Cuna y Nivel Medio. Cada uno de estos niveles se subdivide dependiendo de la edad del menor en: (a) Menores de 1 año: sala cuna menor y sala cuna mixta; (b) niños de 1 año: sala cuna mayor y sala cuna mixta; (c) niños de 2 años: niveles medio menor, medio mixtos, medios y transición y heterogéneo, y (d) niños de 3 años: niveles medio mayor, medio mixtos, medios y transición y heterogéneo. 
cidos y otros en los que existen holguras de capacidad. Es interesante notar que la condición de exceso de matrícula varía según el nivel educacional (vis-à-vis con la edad de los niños), de modo que en promedio las salas cuna tienen $6,61 \%$ de su capacidad sobrepasada por la matrícula efectiva, mientas que los niveles medios presentan una holgura de capacidad equivalente al 4,87\% de su capacidad ${ }^{6}$.

Adicionalmente, nuestro análisis señala la existencia de problemas significativos de institucionalidad en el sector. Por una parte, existe escasa regulación y fiscalización de los centros que ofrecen educación parvularia tanto a nivel de proveedores públicos como privados. Por otro lado, el marco institucional que rige a los proveedores de servicios preescolares es difuso y ha posibilitado que haya institucionalidades específicas a cada proveedor.

Este documento está organizado en seis secciones. En la segunda sección describimos la institucionalidad del sector. En la tercera y cuarta secciones analizamos en forma separada la demanda y oferta por educación preescolar, respectivamente. En la quinta sección realizamos un análisis conjunto de la oferta y la demanda. En la sexta sección, concluimos.

\section{Institucionalidad de la educación preescolar en Chile}

Actualmente es posible identificar cuatro proveedores de educación parvularia a nivel nacional en el segmento de niños de 0 a 3 años: municipalidades, proveedores privados, Junji e Integra.

Junji corresponde a un organismo autónomo vinculado al Ministerio de Educación, creado en 1970 con el propósito de entregar educación integral y de calidad a menores de 4 años en condiciones de vulnerabilidad. Asimismo, está encargado de supervisar y certificar el funcionamiento de otros centros públicos y privados pertenecientes a otras instituciones.

${ }^{6}$ Esta cifra se obtiene comparando la matrícula total y la capacidad declarada para cada nivel educativo (es decir, generando la diferencia porcentual entre ambas cantidades). La capacidad declarada corresponde a la capacidad total por establecimiento y nivel educativo. Se obtiene sumando la capacidad para cada modalidad (sala cuna menor, mayor y mixta, nivel medio menor, mayor y mixto, nivel heterogéneo y medios y transición) que ofrece un establecimiento para cada nivel de enseñanza. La matrícula total corresponde a la suma de la matrícula ofrecida por un determinado centro en todos sus niveles a los cuales puede asistir un menor de acuerdo a su edad. 
Fundación Intrega, corresponde a una fundación privada creada en 1990 a partir de los centros abiertos de la Fundación Nacional de Ayuda a la Comunidad (Funaco), que durante los años ochenta funcionaron como centros asistenciales para niños vulnerables. Actualmente, ofrece programas de educación parvularia para niños entre 84 días y 3 años cuyas familias están en situación de pobreza y vulnerabilidad social.

Los centros municipales corresponden a organismos autónomos, algunos financiados a través de transferencias de fondos desde Junji. Estos últimos corresponden a organismos que operan Vía Transferencia de Fondos (VTF). Esta modalidad requiere la obtención de la certificación de Junji a la infraestructura y programas educativos; quedando sujetos a la supervisión regular de esta institución.

Finalmente, los centros privados corresponden a instituciones financiadas con aportes particulares que deben contar con la autorización del Ministerio de Salud y los permisos municipales correspondientes para entrar en funcionamiento. A diferencia de los otros centros, la certificación de los privados es de carácter voluntaria una vez que los sostenedores de los establecimientos así lo soliciten. El empadronamiento es realizado por Junji, quien además está facultado para realizar visitas periódicas de supervisión a las instalaciones. Debido al carácter voluntario de la certificación de calidad, existe un número desconocido de centros privados que no está registrado en Junji y de los que se desconoce su ubicación, instalaciones y funcionamiento.

Pese a que Junji es la institución encargada de certificar y supervisar la amplia oferta de proveedores de enseñanza preescolar, no cuenta con la capacidad técnica para realizar estas funciones de manera periódica. Tampoco existe un registro unificado de todas las instituciones y centros públicos y privados que ofrecen servicios de educación parvularia. Debido a la inexistencia de este registro tampoco se tiene noción del cumplimiento del currículum educativo ni del grado académico del personal que educa a los menores.

Lo anterior ha posibilitado la existencia de una institucionalidad específica a cada proveedor, en donde Junji e Integra funcionan como dos instituciones sin un vínculo aparente (a pesar de atender a niños de los sectores más vulnerables y ofrecer programas y modalidades alternativas muy similares) y donde existe una importante dimensión privada de la cual se tiene escaso (o nulo) conocimiento. 


\section{Demanda potencial y matrícula en educación parvularia en la Región Metropolitana}

Esta sección busca caracterizar la demanda por educación parvularia en la Región Metropolitana. Para tales efectos consideramos dos fuentes de información: la encuesta Casen 2009 e información proveniente de la FPS. Como veremos a continuación, ambas entregan perspectivas distintas y relevantes de lo que ocurre en este mercado.

En primer lugar, consideramos los datos de la Encuesta Casen $2009^{7}$ como primera aproximación para cuantificar la demanda potencial y efectiva por educación parvularia en la Región Metropolitana ${ }^{8}$. De los resultados de la información contenida en esta encuesta (presentados en la Tabla $\mathrm{N}^{\circ} 1$ ), se observa que en la región existen 355.455 hogares con niños entre 0 y 3 años de edad, que representan un total de 360.957 niños en ese rango de edad. En relación a la asistencia a establecimientos de educación parvularia, la cuarta columna de la Tabla $\mathrm{N}^{\circ} 1$ muestra una asociación positiva entre la edad del niño y la asistencia a salas cuna y jardines infantiles. Así, observamos que los niños de 3 años son quienes registran la mayor tasa de asistencia (cercana a un $42 \%)^{9}$. La Figura $\mathrm{N}^{\circ} 1$ muestra la dependencia administrativa de los centros a los cuales asisten los menores.

Si bien la información de la encuesta Casen es valiosa para comprender los números agregados, no nos permite disponer de información precisa de la demanda por salas cuna y jardines infantiles (ni de la focalización de la oferta). Esto es particularmente importante, pues de acuerdo al artículo 12 de la Ley $20.379^{10}$ — que institucionaliza el Programa Chile Crece Contigo (ChCC) - tanto Junji como Integra deben asegurar

${ }^{7}$ La encuesta de Caracterización Socioeconómica (Casen) 2009 es la encuesta de hogares utilizada para generar los datos oficiales de pobreza y desigualdad de ingresos en Chile. Cuenta con información para 246.924 individuos $\mathrm{y}$ tiene un nivel de representatividad comunal.

${ }^{8}$ La población de la Región Metropolitana representa el 40,22\% del país (noviembre de 2009).

${ }^{9}$ Los resultados presentados en la Tabla $\mathrm{N}^{\circ} 1$ consideran información para niños entre 0 y 3 años en Casen 2009, tabulados según la variable "edad" de la propia encuesta. La cuarta columna de la Tabla $\mathrm{N}^{\circ} 1$ utiliza la información de la pregunta "e3" de la Encuesta Casen 2009: ¿Asiste actualmente a algún establecimiento educacional, jardín infantil, sala cuna $\mathrm{u}$ otro programa preescolar no convencional?

${ }^{10}$ Congreso Nacional de Chile (2009). 
TABLA N ${ }^{\circ}$ 1: $\quad$ NÚMERO DE HOGARES Y NIÑOS ENTRE 0 Y 3 AÑOS DE EDAD QUE ASISTEN A CENTROS DE ENSEÑANZA PARVULARIA EN LA REGIÓN METROPOLITANA SEGÚN CASEN 2009

\begin{tabular}{lrrr}
\hline Edad & Hogares & Niños & Niños que asisten \\
\hline \multirow{2}{*}{ Menores de 1 año } & 82.546 & 84.094 & $3.601(4,28 \%)$ \\
1 año & 79.108 & 80.331 & $8.070(10,05 \%)$ \\
2 años & 102.364 & 104.223 & $20.633(19,79 \%)$ \\
3 años & 91.437 & 92.309 & $38.827(42,06 \%)$ \\
& & & \\
Total & $\mathbf{3 5 5 . 4 5 5}$ & $\mathbf{3 6 0 . 9 5 7}$ & $\mathbf{7 1 . 1 3 1 ( 1 9 , 7 1 \% )}$ \\
& & & \\
\hline
\end{tabular}

Fuente: Casen 2009. Notas: Entre paréntesis se indica el porcentaje de niños que asisten a centros preescolares sobre el total de niños en cada rango de edad.

FIGURA N ${ }^{\circ}$ 1: $\quad$ NÚMERO DE NIÑOS ENTRE 0 Y 3 AÑOS DE EDAD QUE ASISTEN A CENTROS DE ENSEÑANZA PARVULARIA EN LA REGIÓN METROPOLITANA POR DEPENDENCIA ADMINISTRATIVA SEGÚN CASEN 2009

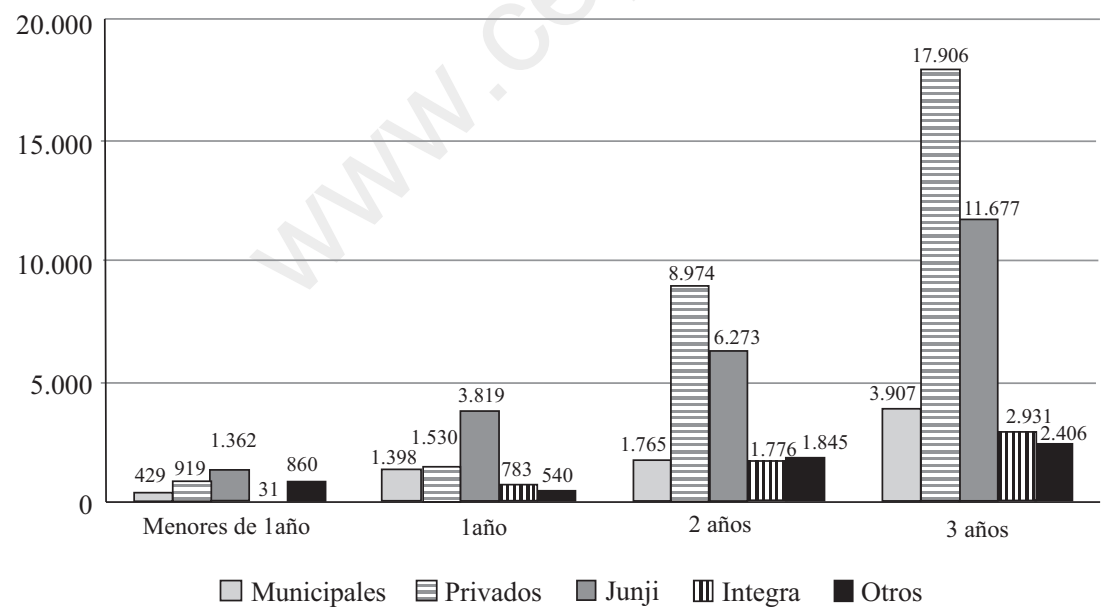

Notas: Municipales agrupa a jardines infantiles administrados por las Corporaciones Municipales y Departamentos Administrativos de Educación Municipal (DAEM). Privados agrupa a establecimientos adminitrados por privados y subvencionados. Otros agrupa a niños que no reportan información sobre la dependencia del establecimiento al que asisten y a aquellos que asisten a centros privados provistos por los empleadores de sus padres.

Fuente: Elaboración propia en base a Casen 2009. 
cupos para aquellos niños de madres que se encuentren en el 60\% más vulnerable de acuerdo a la Ficha de Protección Social (FPS), y que se encuentren estudiando, buscando trabajo o trabajando. Asimismo, la encuesta Casen tampoco entrega información respecto del nivel de vulnerabilidad del hogar que nos permitiría simular los criterios de elegibilidad para acceder a los cupos que entrega Junji e Integra a través de ChCC.

De acuerdo a las consideraciones anteriores, utilizamos datos administrativos de FPS para analizar la forma en que la demanda por educación parvularia (potencial y efectiva) se distribuye en relación a la oferta de centros administrados por Junji e Integra en la Región Metropolitana $^{11}$. La FPS, a diferencia de la encuesta Casen, corresponde a un instrumento de focalización de beneficios sociales. Por este motivo, cuenta con un registro de todos los hogares que se encuentran en situación de vulnerabilidad y que son elegibles para recibir algún tipo de asignación social. Una de las ventajas de utilizar FPS es que permite identificar de mejor manera el grupo objetivo al cual se orienta el programa ChCC y a quienes asisten a Junji e Integra.

Con el objetivo de analizar la forma en que la demanda por educación preescolar (potencial y efectiva) se distribuye en relación a la localización de los establecimientos Junji e Integra (oferta), utilizamos la ubicación geográfica de los hogares (información georeferenciada) disponible en la FPS y de los centros (información pública de la ubicación de centros preescolares) ${ }^{12}$. Esto nos permite responder preguntas relacionadas con el grado de coordinación entre las instituciones públicas para localizar los centros, los factores que explicarían la inasistencia a la enseñanza parvularia (eventualmente asociada a la disponibilidad de los centros en relación a la ubicación de los hogares) y los elementos que caracterizan la decisión de los padres de enviar a sus hijos a centros

${ }^{11}$ Es importante mencionar que la utilización de la información contenida en FPS se llevó a cabo bajo estrictas normas de confidencialidad. Los autores no tuvieron acceso a información que permitiese la individualización de las personas. A junio de 2010 existen 10.906.544 inscritos en FPS. Debido a problemas de duplicidad de datos, la muestra se reduce a 8.047.182 individuos. Del total de individuos a nivel nacional, en la Región Metropolitana existen 130.550 niños entre cero y tres años de edad.

${ }^{12}$ Es relevante notar que cerca de un $90 \%$ de los hogares cuentan con información georeferenciada en la FPS. Para el 10\% restante la inexistencia de esta información se explica por los problemas de digitación en las direcciones y por la falta de mapas actualizados de la Región Metropolitana. 
cercanos a sus hogares o no. A partir de esta información, identificamos como población potencialmente demandante de educación parvularia a todos los niños entre 0 y 3 años de edad inscritos en FPS que residen en la Región Metropolitana. La Tabla $\mathrm{N}^{\circ} 2$ presenta la estadística descriptiva de la información empleada.

En este caso, consideramos como matrícula efectiva a todos los niños entre 0 y 3 años de edad inscritos en FPS y matriculados en Junji e Integra al año 2010 (septiembre y octubre, respectivamente) $)^{13}$. La Tabla $\mathrm{N}^{\circ} 3$ presenta el número de niños entre 0 y 3 años que asisten a centros de educación parvularia según la dependencia del establecimiento a partir de los datos de la encuesta Casen 2009. De ésta se desprende que para todos los niveles, Junji agrupa a la mayoría de los niños matriculados, seguido de los establecimientos particulares (colegios).

TABLA N ${ }^{\circ}$ 2: $\quad$ DEMANDA POTENCIAL POR EDUCACIÓN PARVULARIA EN LA REGIÓN METROPOLITANA SEGÚN FPS Y MATRICULADOS EN JUNJI E INTEGRA A 2010

Demanda potencial: Niños entre 0 y 3 años inscritos en FPS que viven en la Región Metropolitana

Matrícula:

Niños entre 0 y 3 años inscritos en FPS que viven y asisten a centros Junji o Integra en la Región Metropolitana:

Matriculados en Integra (octubre, 2010) 4.472

Notas: (a) Los registros de centros Junji incluyen centros que operan bajo el sistema de transferencias de fondos desde Junji y que son administrados por organizaciones vecinales y/o sin fines de lucro. (b) En esta categoría consideramos a todos los niños entre 0 y 3 años que no aparecen en los registros de matrículas de Junji e Integra. Sin embargo, parte de este grupo podría estar matriculado en centros particulares subvencionados, privados o establecimientos de enseñanza básica y media con dependencias destinadas a la enseñanza preescolar.

Fuente: Ficha de Protección Social e información proporcionada por Junji e Integra.

${ }^{13}$ En los registros de Junji e Integra hay 237.212 niños a nivel país, de los cuales 65.481 están matriculados en centros de la Región Metropolitana. De éstos, 15.972, que tienen entre cero y tres años de edad, están inscritos en FPS. 
TABLA N ${ }^{\circ}$ 3: $\quad$ NIÑOS ENTRE 0 Y 3 AÑOS QUE ASISTEN A LA ENSEÑANZA PARVULARIA SEGÚN LA DEPENDENCIA ADMINISTRATIVA DEL ESTABLECIMIENTO, CASEN 2009

\begin{tabular}{|c|c|c|c|c|c|c|}
\hline \multirow[b]{2}{*}{ Edad } & \multicolumn{6}{|c|}{ Dependencia administrativa } \\
\hline & Municipales $^{(a)}$ & Colegios $^{(b)}$ & Junji & Integra & $\operatorname{Otros}^{(\mathrm{c})}$ & Total \\
\hline Menores de 1 año & $\begin{array}{r}429 \\
(11,91 \%)\end{array}$ & $\begin{array}{r}919 \\
(25,52 \%)\end{array}$ & $\begin{array}{r}1.362 \\
(37,82 \%)\end{array}$ & $\begin{array}{r}31 \\
(0,86 \%)\end{array}$ & $\begin{array}{r}860 \\
(23,88 \%)\end{array}$ & $\begin{array}{r}3.601 \\
(100 \%)\end{array}$ \\
\hline 1 año & $\begin{array}{r}1.398 \\
(17,32 \%)\end{array}$ & $\begin{array}{r}1.530 \\
(18,96 \%)\end{array}$ & $\begin{array}{r}3.819 \\
(47,32 \%)\end{array}$ & $\begin{array}{r}783 \\
(9,70 \%)\end{array}$ & $\begin{array}{r}540 \\
(6,69 \%)\end{array}$ & $\begin{array}{r}8.070 \\
(100 \%)\end{array}$ \\
\hline 2 años & $\begin{array}{r}1.765 \\
(8,55 \%)\end{array}$ & $\begin{array}{r}8.974 \\
(43,49 \%)\end{array}$ & $\begin{array}{r}6.273 \\
(30,40 \%)\end{array}$ & $\begin{array}{r}1.776 \\
(8,61 \%)\end{array}$ & $\begin{array}{r}1.845 \\
(8,94 \%)\end{array}$ & $\begin{array}{l}20.633 \\
(100 \%)\end{array}$ \\
\hline 3 años & $\begin{array}{r}3.907 \\
(10,06 \%)\end{array}$ & $\begin{array}{r}17.906 \\
(46,12 \%)\end{array}$ & $\begin{array}{r}11.677 \\
(30,07 \%)\end{array}$ & $\begin{array}{r}2.931 \\
(7,55 \%)\end{array}$ & $\begin{array}{r}2.406 \\
(6,20 \%)\end{array}$ & $\begin{array}{r}38.827 \\
(100 \%)\end{array}$ \\
\hline Total & $\begin{array}{r}7.499 \\
(10,54 \%)\end{array}$ & $\begin{array}{r}29.329 \\
(41,23 \%)\end{array}$ & $\begin{array}{r}23.132 \\
(32,52 \%)\end{array}$ & $\begin{array}{r}5.521 \\
(7,76 \%)\end{array}$ & $\begin{array}{r}5.651 \\
(7,94 \%)\end{array}$ & $\begin{array}{r}71.133 \\
(100 \%)\end{array}$ \\
\hline
\end{tabular}

Notas: (a) Municipales agrupa a las Corporaciones Municipales y Departamentos Administrativos de Educación Municipal (DAEM). (b) Colegios agrupa a establecimientos particulares privados y subvencionados. (c) Otros agrupa a niños que no reportan información sobre la dependencia del establecimiento al que asisten y a aquellos que asisten a centros privados provistos por los empleadores de sus padres.

Fuente: Elaboración propia en base a Casen 2009.

La Figura $\mathrm{N}^{\circ} 2$ presenta la distribución de la demanda potencial y efectiva en la Región Metropolitana utilizando datos de la Encuesta Casen 2009 y de FPS. En este caso, hemos dividido la región en cuatro sectores, tomando como punto de referencia la Plaza de Armas de Santiago, lugar considerado el centro de la ciudad ${ }^{14}$. De la figura podemos observar que FPS reproduce correctamente la distribución de niños entre 0 y 3 años que se observa en los datos agregados provenientes de Casen 2009.

Lo anterior es interesante debido a las características de la población en ambas muestras. Tal como mencionamos anteriormente, Casen 2009 constituye una muestra representativa a nivel nacional mientras que FPS agrupa a familias que han decidido entregar la información necesaria para el cálculo de su condición de vulnerabilidad. Luego, es plausible esperar que la distribución geográfica de ambas muestras di-

14 Debido a la existencia de comunas que intersectan más de un sector simultáneamente, hemos imputado la población total tanto de Casen 2009 como de FPS al sector que agrupe a más del $85 \%$ de la población total de la comuna. 


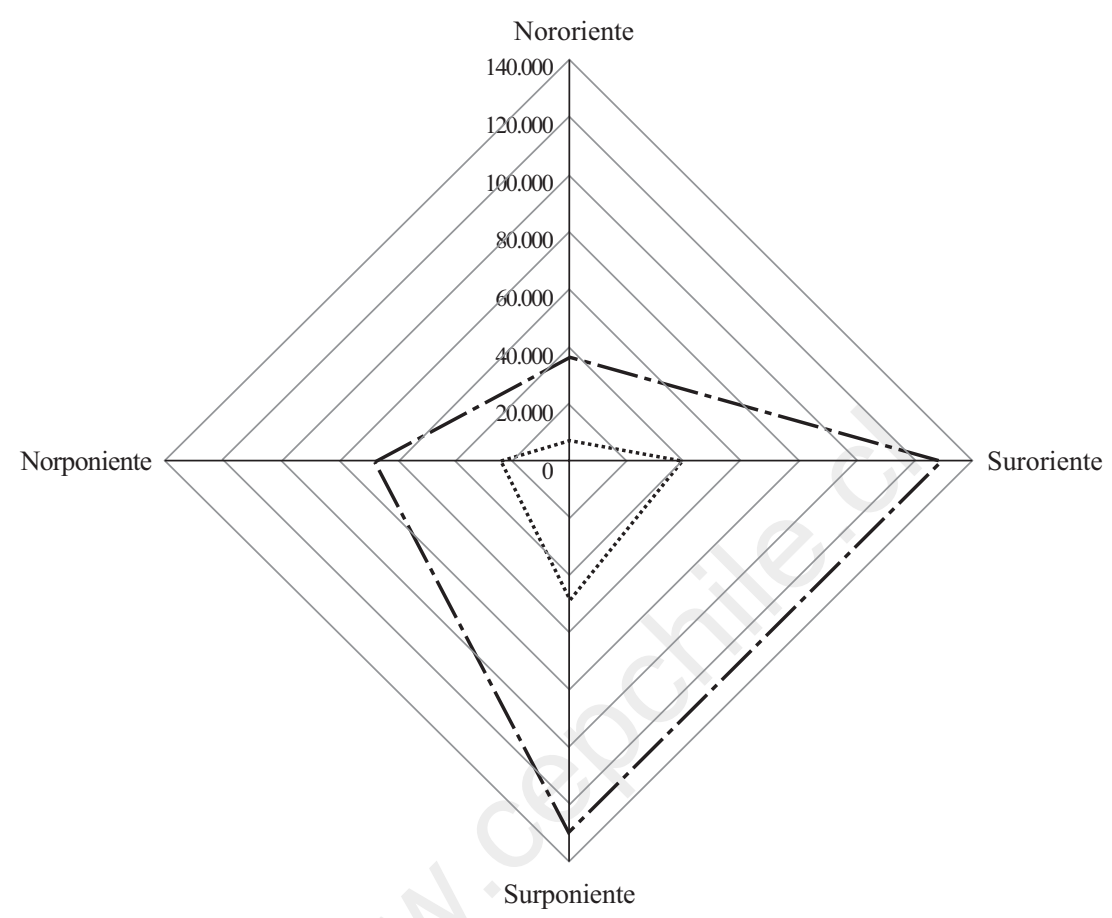

-- - FPS - Casen 2009

Notas: (a) Nororiente: Alhué, Huechuraba, Las Condes, Lo Barnechea, Recoleta y Vitacura. (b) Suroriente: La Florida, La Granja, La Pintana, La Reina, Macul, Ñuñoa, Peñalolén, Pirque, Providencia, Puente Alto, San Joaquín, San José de Maipo, San Miguel y San Ramón. (c) Buin, Calera de Tango, Cerrillos, El Bosque, El Monte, Estación Central, Isla de Maipo, La Cisterna, Lo Espejo, Lo Prado, Maipú, María Pinto, Melipilla, Padre Hurtado, Paine, Pedro Aguirre Cerda, Peñaflor, Pudahuel, San Bernardo, Talagante y Tiltil. (d) Cerro Navia, Colina, Conchalí, Curacaví, Independencia, Lampa, Quilicura, Quinta Normal y Renca.

Fuente: Elaboración propia en base a Casen 2009 y Ficha de Protección Social.

fiera. Sin embargo, observamos que FPS reproduce la forma en que se distribuye la población en la Región Metropolitana, a una menor escala.

Otro aspecto interesante de resaltar, es que gran parte de la población potencialmente demandante de educación preescolar se ubica en el sector sur de la ciudad (situación consistente en Casen y FPS).

Del universo de niños entre 0 y 3 años inscritos en FPS que viven en la Región Metropolitana, la Tabla $N^{\circ} 4$ presenta el número de niños 
TABLA N ${ }^{\circ}$ 4: $\quad$ NÚMERO DE NIÑOS ENTRE 0 Y 3 AÑOS INSCRITOS EN FPS SEGÚN SU SITUACIÓN DE MATRÍCULA EN JUNJI E INTEGRA A 2010

\begin{tabular}{cccc}
\hline Edad & $\begin{array}{c}\text { Matriculados } \\
\text { en Junji e Integra }\end{array}$ & No matriculados & Total \\
\hline \multirow{2}{*}{ Menores de 1 año } & 226 & 8.754 & 8.980 \\
& $(2,52 \%)$ & $(97,48 \%)$ & $(100 \%)$ \\
1 año & 2.708 & 29.250 & 31.958 \\
& $(8,47 \%)$ & $(91,53 \%)$ & $(100 \%)$ \\
2 años & 5.261 & 32.686 & 37.947 \\
3 años & $(13,86 \%)$ & $(86,14 \%)$ & $(100 \%)$ \\
& 7.777 & 31.087 & 38.864 \\
Total & $(20,01 \%)$ & $(79,99 \%)$ & $(100 \%)$ \\
& & & $\mathbf{1 1 7 . 7 4 9}$ \\
& $\mathbf{1 5 . 9 7 2}$ & $\mathbf{1 0 1 . 7 7 7}$ & $\mathbf{( 1 0 0 \% )}$ \\
\hline
\end{tabular}

Notas: Debido a que disponemos de registros en FPS hasta junio de 2010, el número de niños menores de 1 año es relativamente más bajo en relación a los niños de 1 a 3 años. Entre paréntesis se indica el porcentaje de niños que asisten y no asisten a centros preescolares sobre el total de niños en cada rango de edad.

Fuente: Ficha de Protección Social (FPS) e información proporcionada por Junji e Integra.

matriculados en jardines infantiles y salas cunas pertenecientes a Junji e Integra. Los resultados muestran una tendencia similar a la observada a partir de los datos Casen 2009. A medida que se incrementa la edad, aumenta la matrícula. Esto aunque la magnitud de los valores difiere de la obtenida en la encuesta Casen (lo cual es absolutamente plausible considerando que no disponemos de registros de niños matriculados en otros establecimientos, ya sea colegios, jardines y salas cuna privados).

En relación a la distribución geográfica de la matrícula, la Tabla $\mathrm{N}^{\circ} 5$ presenta el número de niños matriculados y no matriculados según sector de residencia. Los resultados indican que las comunas del sector nororiente de la región alcanzan el porcentaje mayor de asistencia relativo a la cantidad de niños inscritos en FPS en la comuna (equivalente a un $18,77 \%$ ), mientras que las comunas rurales y del sector surponiente alcanzan los porcentajes más bajos de la región (equivalentes a 12,01\% y $12,67 \%$ respectivamente). 
TABLA N ${ }^{\circ}$ 5: $\quad$ DISTRIBUCIÓN DE LA MATRÍCULA EN ESTABLECIMIENTOS JUNJI E INTEGRA SEGÚN SECTORES DE LA REGIÓN METROPOLITANA

\begin{tabular}{|c|c|c|c|c|}
\hline Zona & Sector & Matriculados & No matriculados & Total \\
\hline \multirow{10}{*}{ Urbana } & Norte $^{(a)}$ & $\begin{array}{c}1.797 \\
(13,67 \%)\end{array}$ & $\begin{array}{c}11.353 \\
(86.33 \%)\end{array}$ & $\begin{array}{l}13.150 \\
(100 \%)\end{array}$ \\
\hline & Nororiente $^{(b)}$ & $\begin{array}{c}706 \\
(18,77 \%)\end{array}$ & $\begin{array}{c}3.055 \\
(81,23 \%)\end{array}$ & $\begin{array}{c}3.761 \\
(100 \%)\end{array}$ \\
\hline & Norponiente $^{(c)}$ & $\begin{array}{c}2.375 \\
(13,13 \%)\end{array}$ & $\begin{array}{c}15.709 \\
(86,87 \%)\end{array}$ & $\begin{array}{l}18.084 \\
(100 \%)\end{array}$ \\
\hline & \multirow[t]{2}{*}{ Centro ${ }^{(d)}$} & 437 & 2.786 & 3.223 \\
\hline & & $(13,56 \%)$ & $(86,44 \%)$ & $(100 \%)$ \\
\hline & Suroriente ${ }^{(\mathrm{e})}$ & $\begin{array}{c}3.157 \\
(15,13 \%)\end{array}$ & $\begin{array}{c}17.705 \\
(84,87 \%)\end{array}$ & $\begin{array}{l}20.862 \\
(100 \%)\end{array}$ \\
\hline & Surponiente ${ }^{(\mathrm{f})}$ & $\begin{array}{c}1.471 \\
(12,67 \%)\end{array}$ & $\begin{array}{c}10.143 \\
(87,33 \%)\end{array}$ & $\begin{array}{c}11.614 \\
(100 \%)\end{array}$ \\
\hline & $\operatorname{Sur}^{(g)}$ & $\begin{array}{c}3.345 \\
(13,54 \%)\end{array}$ & $\begin{array}{c}21.365 \\
(86,46 \%)\end{array}$ & $\begin{array}{l}24.710 \\
(100 \%)\end{array}$ \\
\hline & Rural $^{(\mathrm{h})}$ & $\begin{array}{c}2.684 \\
(12,01 \%)\end{array}$ & $\begin{array}{c}19.661 \\
(87,99 \%)\end{array}$ & $\begin{array}{l}22.345 \\
(100 \%)\end{array}$ \\
\hline & Total & $\begin{array}{c}15.972 \\
(13,56 \%)\end{array}$ & $\begin{array}{c}101.777 \\
(86,44 \%)\end{array}$ & $\begin{array}{l}117.749 \\
(100 \%)\end{array}$ \\
\hline
\end{tabular}

Notas: (a) Agrupa a Conchalí, Huechuraba, Independencia, Quilicura y Recoleta. (b) Agrupa a La Reina, Las Condes, Lo Barnechea, Nuñoa, Providencia y Vitacura. (c) Agrupa a Cerro Navia, Lo Prado, Pudahuel, Quinta Normal y Renca. (d) Agrupa a Santiago. (e) La Florida, Macul, Peñalolén y Puente Alto. (f) Agrupa a Cerrillos, Estación Central y Maipú. (g) Agrupa a El Bosque, La Cisterna, La Granja, La Pintana, Lo Espejo, Pedro Aguirre Cerda, San Joaquín, San Miguel y San Ramón. (h) Agrupadas en base a su porcentaje de ruralidad, incluye a Alhué, Buin, Calera de Tango, Colina, Curacaví, El Monte, Isla de Maipo, Lampa, María Pinto, Melipilla, Padre Hurtado, Paine, Peñaflor, Pirque, San Bernardo, San Pedro, San José de Maipo, Tiltil y Talagante. Entre paréntesis se indica el porcentaje de niños que asisten y no asisten a salas cunas y jardines infantiles sobre el total de niños que viven en cada sector de la Región Metropolitana.

Fuente: Ficha de Protección Social (FPS) e información proporcionada por Junji e Integra.

La Figura $\mathrm{N}^{\circ} 3$ presenta la distribución de la matrícula en Junji e Integra para niños entre 0 y 3 años inscritos en FPS por edad y sector de la Región Metropolitana. De la figura observamos que la matrícula se incrementa conforme aumenta la edad de los niños y que es nuevamente el sector sur (surponiente y suroriente) el que presenta los niveles de matrícula más altos. 
FIGURA ${ }^{\circ}$ 3: $\quad$ NÚMERO DE NIÑOS MATRICULADOS (0-3 AÑOS) EN JUNJI E INTEGRA POR EDAD Y SECTOR DE LA REGIÓN METROPOLITANA

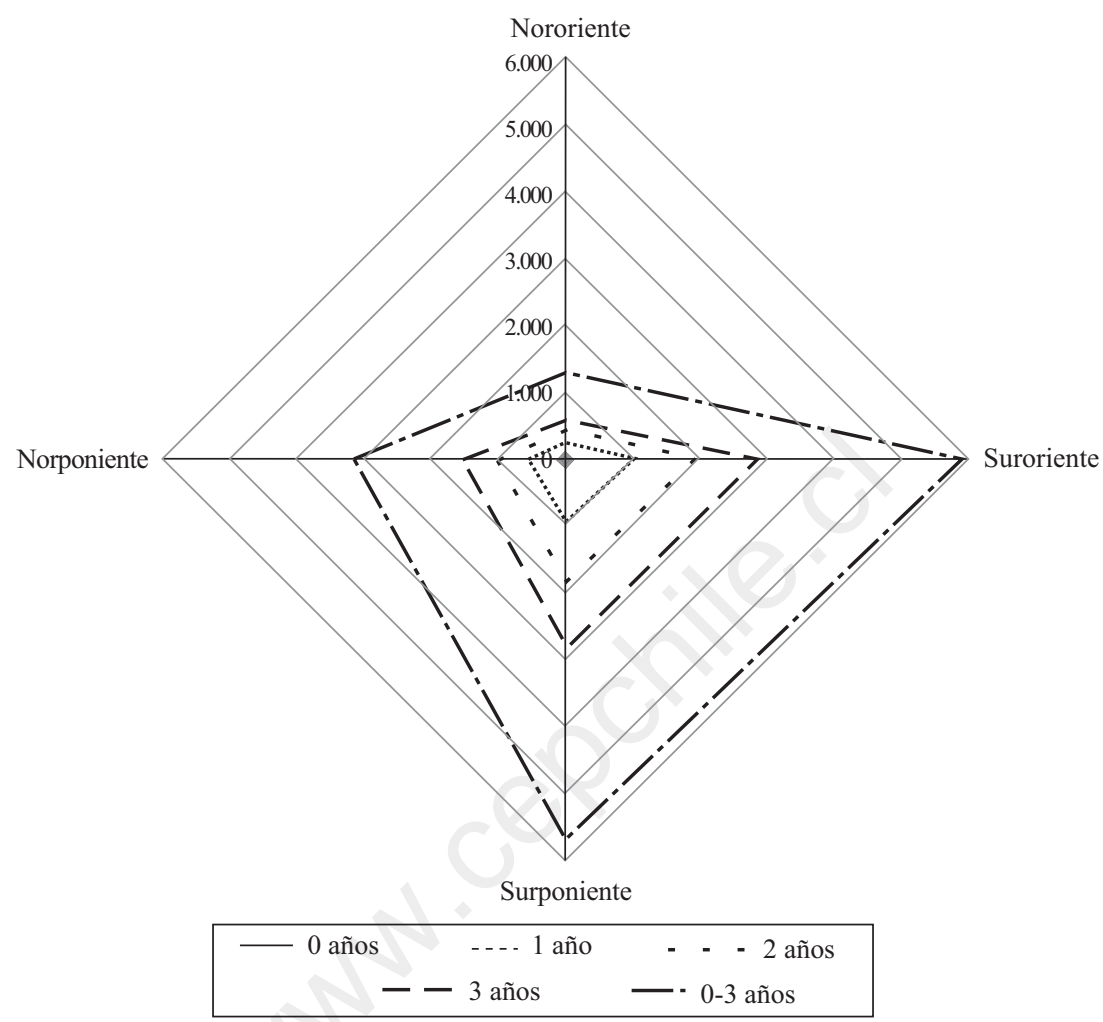

Notas: Para mayores detalles sobre la descripción de las comunas que componen cada sector, ver nota de la Figura $\mathrm{N}^{\circ} 2$.

Fuente: Elaboración propia en base a la Ficha de Protección Social (FPS) e información proporcionada por Junji e Integra.

\subsection{Distancia hasta la sala cuna o jardín infantil como determinante de la decisión de matrícula}

Como anticipamos anteriormente, un elemento importante en el contexto de los determinantes de la demanda por salas cuna y jardines infantiles es su disponibilidad y distribución geográfica. En esta sección exploramos este elemento en detalle. En particular, nos preguntamos en qué medida la oferta disponible de centros y las características de éstos, posibilitan que los niños asistan a establecimientos ubicados en la misma comuna de residencia. 
La Tabla $\mathrm{N}^{\circ} 6$ muestra el número de niños que asiste a centros ubicados en la misma comuna de residencia, en una comuna distinta en la Región Metropolitana y aquellos que a pesar de tener su domicilio en la Región Metropolitana (según FPS) se encuentran matriculados en otra región. Mediante este ejercicio intentamos aproximar el grado de movilidad de los niños respecto del sector en que viven y del que se encuentran ubicados los establecimientos a los que asisten.

Los resultados anteriores indican que sobre el $70 \%$ de los niños entre 0 y 3 años asisten a centros de Junji e Integra ubicados en la misma comuna de residencia, cifra que se mantiene constante al desagregarla por edad. En primera instancia, esto sugiere que los padres prefieren centros cercanos a sus hogares para enviar a sus hijos. De ser así, la distancia entre el hogar-centro sería un factor importante en la decisión de envío y posteriormente en la elección final del centro al que asisten los menores.

La Tabla $\mathrm{N}^{\circ} 7$ muestra la distancia promedio entre los hogares de los niños matriculados en Junji e Integra y los centros a los que asisten, desagregada por la edad del menor, dependencia administrativa de éste y la localización de su comuna de residencia. Los resultados indican que, en promedio, la distancia hogar-centro es similar entre niños meno-

TABLA N ${ }^{\circ}$ 6: $\quad$ NÚMERO DE NIÑOS QUE ESTÁN MATRICULADOS EN LA MISMA COMUNA DE RESIDENCIA O BIEN FUERA DE ÉSTA SEGÚN EDAD

\begin{tabular}{ccccc}
\hline Edad & $\begin{array}{c}\text { Vive en RM y } \\
\text { asiste a la misma } \\
\text { comuna de RM }\end{array}$ & $\begin{array}{c}\text { Vive en RMy } \\
\text { asiste a una comuna } \\
\text { distinta de RM }\end{array}$ & $\begin{array}{c}\text { Vive en RM y } \\
\text { asiste a otra comuna } \\
\text { fuera de RM }\end{array}$ & Total \\
Menores de 1 año & 172 & & & \\
\multirow{2}{*}{1 año } & $(76,11 \%)$ & $(22,57 \%)$ & $(1,33 \%)$ & $(100 \%)$ \\
& 1.966 & 653 & 89 & 2.708 \\
2 años & $(72,60 \%)$ & $(24,11 \%)$ & $(3,29 \%)$ & $(100 \%)$ \\
\multirow{2}{*}{3 años } & 3.838 & 1.256 & 167 & 5.261 \\
& $(72,95 \%)$ & $(23,87 \%)$ & $(3,17 \%)$ & $(100 \%)$ \\
Total & 5.767 & 1.789 & 221 & 7.777 \\
& $(74,15 \%)$ & $(23,00 \%)$ & $(2,84 \%)$ & $(100 \%)$ \\
& $\mathbf{1 1 . 7 4 3}$ & $\mathbf{3 . 7 4 9}$ & $\mathbf{4 8 0}$ & $\mathbf{1 5 . 9 7 2}$ \\
& $\mathbf{( 7 3 , 5 2 \% )}$ & $\mathbf{( 2 3 , 4 7 \% )}$ & $\mathbf{( 3 , 0 1 \% )}$ & $\mathbf{( 1 0 0 \% )}$ \\
\hline
\end{tabular}

Fuente: Elaboración propia en base a la Ficha de Protección Social (FPS) e información proporcionada por Junji e Integra. 
TABLA N ${ }^{\circ}$ 7: $\quad$ DISTANCIA PROMEDIO (KILÓMETROS) HOGAR-CENTRO DE NIÑOS ENTRE 0 Y 3 AÑOS INSCRITOS EN FPS Y MATRICULADOS EN JUNJI E INTEGRA AL AÑO 2010

\begin{tabular}{|c|c|c|c|c|c|c|}
\hline \multirow[t]{2}{*}{ Edad } & \multicolumn{3}{|c|}{$\begin{array}{l}\text { Matriculados en la misma comuna } \\
\text { de residencia }\end{array}$} & \multicolumn{3}{|c|}{$\begin{array}{l}\text { Matriculados en una comuna } \\
\text { distinta a la de residencia }\end{array}$} \\
\hline & Junji & Integra & Todos & Junji & Integra & Todos \\
\hline Menores de 1 año & 2,392 & 1,385 & 2,262 & 4,003 & 7,044 & 4,584 \\
\hline 1 año & 1,893 & 1,715 & 1,859 & 5,587 & 5,013 & 5,461 \\
\hline 2 años & 1,951 & 1,791 & 1,913 & 5,329 & 8,472 & 6,099 \\
\hline 3 años & 2,420 & 2,108 & 2,321 & 5,957 & 5,421 & 5,771 \\
\hline Distancia promedio & 2,164 & 1,750 & 2,089 & 5,219 & 6,488 & 5,479 \\
\hline
\end{tabular}

Notas: Todas las distancias se expresan en kilómetros. En este caso, la tabulación se efectúa únicamente para el grupo de niños entre 0 y 3 años que están matriculados en la Región Metropolitana y para quienes se tienen las ubicaciones georeferenciadas de los establecimientos en que están matriculados. Es decir, del total de niños que viven en la Región Metropolitana y asisten a jardines infantiles Junji e Integra (15.972), 15.492 viven y asisten a establecimientos en la Región Metropolitana y para 15.393 se tienen las direcciones georeferenciadas de sus establecimientos.

Fuente: Elaboración propia en base a la Ficha Protección Social (FPS) e información proporcionada por Junji e Integra.

res de 1 año y niños de 3 años cuyos centros están ubicados en la misma comuna de residencia ${ }^{15}$. Asimismo, en promedio, para niños matriculados en centros ubicados en la misma comuna de residencia la distancia hogar-centro es menor para niños matriculados en centros Integra en comparación a aquellos que asisten a centros Junji. Lo contrario ocurre en el caso de niños matriculados en una comuna distinta a la comuna de residencia.

${ }^{15}$ A partir de las coordenadas geográficas de los hogares y establecimientos calculamos las distancias utilizando la siguiente fórmula. Sean A y B dos ubicaciones distintas asociadas a las coordenadas (lat(A), lon(A)) y (lat(B), lon(B)), respectivamente. Luego, la distancia entre ambos puntos viene dada por (Sinnott, 1984):

$$
\begin{aligned}
& \tau(A, B)=\left(\frac{\sin (\operatorname{lat}(B)-\operatorname{lat}(A))}{2}\right)^{2}+\cos (\operatorname{lat}(A)) \cdot \cos (\operatorname{lat}(B)) \cdot\left(\frac{\sin (\operatorname{lon}(B)-\operatorname{lon}(A))}{2}\right)^{2} \\
& d(A, B)=6.367 \cdot(2 \cdot \arctan (\sqrt{\tau(A, B}), \sqrt{1-\tau(A, B)}))
\end{aligned}
$$

Donde $d(A, B)$ corresponde a la distancia entre los puntos A y B expresada en kilómetros. 
Extendiendo el análisis anterior a todo el grupo de potenciales demandantes de educación parvularia (es decir, a todos los niños entre 0 y 3 años inscritos en FPS para quienes se cuenta con la información georeferenciada de sus hogares), la Tabla $N^{\circ} 8$ muestra las distancias promedio desde el hogar al centro más cercano (independiente de si el niño está matriculado), diferenciadas según la edad del menor y su situación de matrícula en Junji e Integra. En este caso, la asignación de los establecimientos más cercanos, se efectuó respetando la oferta existente para cada edad. En otras palabras, consideramos el conjunto de centros que ofrecen los niveles a los que pueden asistir los menores de acuerdo a su edad ${ }^{16}$. De esta forma, dentro del conjunto de centros que ofrecen los niveles para cada edad, se escogió el más cercano al hogar del menor.

TABLA N ${ }^{\circ}$ 8: $\quad$ DISTANCIA HOGAR-CENTRO MÁS CERCANO (KILÓMETROS) SEGÚN SITUACIÓN DE MATRÍCULA EN JUNJI E INTEGRA

\begin{tabular}{ccccc}
\hline \multirow{2}{*}{ Edad } & No matriculados & \multicolumn{2}{c}{ Matriculados } & Todos \\
\cline { 3 - 3 } & & $\begin{array}{c}\text { Viven y asisten } \\
\text { a la misma } \\
\text { comuna de RM }\end{array}$ & $\begin{array}{c}\text { Viven en RM y } \\
\text { asisten a una comuna } \\
\text { distinta en RM }\end{array}$ & \\
\hline Menores de 1 año & 0,814 & 0,742 & 0,69 & 0,812 \\
1 año & 0,781 & 0,51 & 0,563 & 0,794 \\
2 años & 0,982 & 0,574 & 0,584 & 0,929 \\
3 años & 0,707 & 1,06 & 0,461 & 0,748 \\
Distancia promedio & $\mathbf{0 , 8 2 1}$ & $\mathbf{0 , 7 2 2}$ & $\mathbf{0 , 5 7 5}$ & $\mathbf{0 , 8 2 1}$ \\
\hline
\end{tabular}

Fuente: Elaboración propia en base a la Ficha de Protección Social (FPS) e información proporcionada por Junji e Integra.

De los resultados de la Tabla $\mathrm{N}^{\circ} 8$, se observa que, en promedio, la distancia hogar-centro más cercano es menor para niños matriculados en relación a los no matriculados. Además, la distancia promedio hogarcentro más cercano es menor que la distancia efectiva entre el hogar y el establecimiento al que asisten los niños matriculados en la misma comuna de residencia (tercera columna de la Tabla $\mathrm{N}^{\circ} 8$ y cuarta columna de la Tabla $\mathrm{N}^{\circ} 7$, respectivamente).

${ }^{16}$ Los niveles asignados de acuerdo a la edad corresponden a: (a) Menores de 1 año: sala cuna menor y sala cuna mixta; (b) Niños de 1 año: sala cuna mayor y sala cuna mixta; (c) Niños de 2 años: niveles medio menor, medio mixtos, medios y transición y heterogéneo y (d) Niños de 3 años: niveles medio mayor, medio mixtos, medios y transición y heterogéneo. 
Las Tablas Nos. 9A y 9B presentan el porcentaje de niños matriculados y no matriculados, respectivamente, cuya distancia hogar-centro más cercano se encuentra inscrita en distintos radios de distancia. A tra-

TABLA N ${ }^{\circ}$ 9A: $\quad$ PORCENTAJE DE NIÑOS MATRICULADOS EN JUNJI O INTEGRA CUYA DISTANCIA HOGAR-CENTRO MÁS CERCANO (KILÓMETROS) SE ENCUENTRA INSCRITA EN DISTINTOS RADIOS DE DISTANCIA

\begin{tabular}{|c|c|c|c|c|c|c|}
\hline \multirow[t]{2}{*}{ Edad } & \multicolumn{5}{|c|}{ Distancia hogar-centro más cercano (radios en kilómetros) } & \multirow[t]{2}{*}{ Total } \\
\hline & Menos de $1 \mathrm{~km}$ & 1 a $2 \mathrm{kms}$ & 2 a $3 \mathrm{kms}$ & 3 a $4 \mathrm{kms}$ & más de $4 \mathrm{kms}$ & \\
\hline Menores de 1 año & $\begin{array}{c}206 \\
(91,15 \%)\end{array}$ & $\begin{array}{c}14 \\
(6,19 \%)\end{array}$ & $\begin{array}{c}1 \\
(0,44 \%)\end{array}$ & $\begin{array}{c}1 \\
(0,44 \%)\end{array}$ & $\begin{array}{c}4 \\
(1,77 \%)\end{array}$ & $\begin{array}{c}226 \\
(100 \%)\end{array}$ \\
\hline 1 año & $\begin{array}{c}2.523 \\
(93,17 \%)\end{array}$ & $\begin{array}{c}133 \\
(4,91 \%)\end{array}$ & $\begin{array}{c}17 \\
(0,63 \%)\end{array}$ & $\begin{array}{c}8 \\
(0,30 \%)\end{array}$ & $\begin{array}{c}27 \\
(1,00 \%)\end{array}$ & $\begin{array}{c}2.708 \\
(100 \%)\end{array}$ \\
\hline 2 años & $\begin{array}{c}4.866 \\
(92,49 \%)\end{array}$ & $\begin{array}{c}262 \\
(4,98 \%)\end{array}$ & $\begin{array}{c}60 \\
(1,14 \%)\end{array}$ & $\begin{array}{c}19 \\
(0,36 \%)\end{array}$ & $\begin{array}{c}54 \\
(1,03 \%)\end{array}$ & $\begin{array}{c}5.261 \\
(100 \%)\end{array}$ \\
\hline 3 años & $\begin{array}{c}7.167 \\
(92,16 \%)\end{array}$ & $\begin{array}{c}437 \\
(5,62 \%)\end{array}$ & $\begin{array}{c}74 \\
(0,95 \%)\end{array}$ & $\begin{array}{c}26 \\
(0,33 \%)\end{array}$ & $\begin{array}{c}73 \\
(0,94 \%)\end{array}$ & $\begin{array}{c}7.777 \\
(100 \%)\end{array}$ \\
\hline Total & $\begin{array}{c}14.762 \\
(92,42 \%)\end{array}$ & $\begin{array}{c}846 \\
(5,30 \%)\end{array}$ & $\begin{array}{c}152 \\
(0,95 \%)\end{array}$ & $\begin{array}{c}54 \\
(0,34 \%)\end{array}$ & $\begin{array}{c}158 \\
(0,99 \%)\end{array}$ & $\begin{array}{c}15.972 \\
(100 \%)\end{array}$ \\
\hline
\end{tabular}

Fuente: Elaboración propia en base a la Ficha de Protección Social (FPS) e información proporcionada por Junji e Integra.

TABLA No 9B: $\quad$ PORCENTAJE DE NIÑOS NO MATRICULADOS CUYA DISTANCIA HOGAR-CENTRO MÁS CERCANO (KILÓMETROS) SE ENCUENTRA INSCRITA EN RADIOS DE DISTANCIA

\begin{tabular}{|c|c|c|c|c|c|c|}
\hline \multirow[t]{2}{*}{ Edad } & \multicolumn{5}{|c|}{ Distancia hogar-centro más cercano (radios en kilómetros) } & \multirow[t]{2}{*}{ Total } \\
\hline & Menos de $1 \mathrm{~km}$ & 1 a $2 \mathrm{kms}$ & 2 a $3 \mathrm{kms}$ & 3 a $4 \mathrm{kms}$ & más de 4 kms & \\
\hline Menores de 1 año & $\begin{array}{c}7.509 \\
(85,78 \%)\end{array}$ & $\begin{array}{c}900 \\
(10,28 \%)\end{array}$ & $\begin{array}{c}187 \\
(2,14 \%)\end{array}$ & $\begin{array}{c}36 \\
(0,41 \%)\end{array}$ & $\begin{array}{c}122 \\
(1,39 \%)\end{array}$ & $\begin{array}{c}8.754 \\
(100 \%)\end{array}$ \\
\hline 1 año & $\begin{array}{c}26.081 \\
(89,17 \%)\end{array}$ & $\begin{array}{c}2.326 \\
(7,95 \%)\end{array}$ & $\begin{array}{c}346 \\
(1,18 \%)\end{array}$ & $\begin{array}{c}111 \\
(0,38 \%)\end{array}$ & $\begin{array}{c}386 \\
(1,32 \%)\end{array}$ & $\begin{array}{l}29.250 \\
(100 \%)\end{array}$ \\
\hline 2 años & $\begin{array}{c}29.016 \\
(88,77 \%)\end{array}$ & $\begin{array}{c}2.759 \\
(8,44 \%)\end{array}$ & $\begin{array}{c}434 \\
(1,33 \%)\end{array}$ & $\begin{array}{c}110 \\
(0,34 \%)\end{array}$ & $\begin{array}{c}367 \\
(1,12 \%)\end{array}$ & $\begin{array}{c}32.686 \\
(100 \%)\end{array}$ \\
\hline 3 años & $\begin{array}{c}27.051 \\
(87,02 \%)\end{array}$ & $\begin{array}{c}3.069 \\
(9,87 \%)\end{array}$ & $\begin{array}{c}441 \\
(1,42 \%)\end{array}$ & $\begin{array}{c}138 \\
(0,44 \%)\end{array}$ & $\begin{array}{c}388 \\
(1,25 \%)\end{array}$ & $\begin{array}{c}31.087 \\
(100 \%)\end{array}$ \\
\hline Total & $\begin{array}{c}89.657 \\
(88,09 \%)\end{array}$ & $\begin{array}{c}9.054 \\
(8,90 \%)\end{array}$ & $\begin{array}{c}1.408 \\
(1,38 \%)\end{array}$ & $\begin{array}{c}395 \\
(0,39 \%)\end{array}$ & $\begin{array}{c}1.263 \\
(1,24 \%)\end{array}$ & $\begin{array}{l}101.777 \\
(100 \%)\end{array}$ \\
\hline
\end{tabular}

Fuente: Elaboración propia en base a la Ficha de Protección Social (FPS) e información proporcionada por Junji e Integra. 
vés de este ejercicio, intentamos aproximar la distribución geográfica de los niños en relación a la oferta disponible de centros preescolares para rangos discretos de distancia y edades de los menores. De los resultados, se observa que en promedio sobre el $90 \%$ de los niños entre 0 y 3 años disponen de un centro a menos de 1 kilómetro de su hogar (asignado según la edad del menor y el nivel educativo al que le corresponde asistir). Más interesante aún, este porcentaje estaría diferenciado por la condición de matrícula de los menores. De este modo, sobre el 90\% de los matriculados en Junji e Integra tendría un centro a menos de 1 kilómetro de su hogar, mientras que sobre el $88 \%$ de los no matriculados estaría en la misma situación.

La Tabla $\mathrm{N}^{\circ} 10$ compara el número de niños que asisten al centro más cercano a su hogar en relación al número de niños que asiste a un centro distinto ${ }^{17}$. La hipótesis detrás de este ejercicio es que si efectivamente la distancia entre el hogar y el centro importa en la decisión de envío y luego en la elección del centro, los padres debiesen enviar a sus hijos al centro más cercano al hogar, el cual además —en base a los resultados de la Tabla $\mathrm{N}^{\circ} 7$ - estaría restringido a la comuna de residencia. Sin embargo, de la Tabla $\mathrm{N}^{\circ} 10$ se observa que sólo el 30,31\% de los niños asiste al establecimiento más cercano mientras que el 69,69\% de los niños matriculados entre 0 y 3 años asiste a otros establecimientos.

Este resultado no descarta la hipótesis de que la distancia pueda ser una variable importante en la elección del centro al que asiste el menor. Si bien los padres parecen no escoger el centro más cercano al hogar, escogen un establecimiento ubicado en un radio de distancia próximo al hogar; donde este margen de proximidad vendría dado por el área donde viven (en este caso la comuna de residencia) y que eventualmente podrían ser otras características de los hogares o de los centros las que determinan finalmente la elección del centro al que asisten sus hijos ${ }^{18}$.

${ }^{17}$ Considerando el grupo de niños que está matriculado en comunas de la Región Metropolitana (esto explica la reducción en el tamaño muestral en 480 observaciones correspondientes a los niños que viven en la Región Metropolitana pero que asisten a establecimientos ubicados fuera de ésta).

18 Otra lectura de este resultado dice relación con la importancia de la obligación de las empresas de proveer salas cuna en caso de contar con más de 20 trabajadoras. Esto podría explicar el porcentaje de niños que no asisten a la sala cuna más cercana. 
TABLA N ${ }^{\circ}$ 10: $\quad$ COMPARACIÓN ENTRE LA ASISTENCIA EFECTIVA Y LA ASISTENCIA AL CENTRO MÁS CERCANO PARA NIÑOS INSCRITOS EN FPS Y MATRICULADOS EN JUNJI O INTEGRA

\begin{tabular}{lcc}
\hline Categoría & Observaciones & $\%$ \\
\hline & & \\
Asisten al centro más cercano & 4.695 & $30,31 \%$ \\
Asisten a otro centro & 10.797 & $69,69 \%$ \\
Total & $\mathbf{1 5 . 4 9 2}$ & $\mathbf{1 0 0 \%}$ \\
\hline
\end{tabular}

Notas: En este caso, la muestra de observaciones disminuye en 480 observaciones debido a que se trata de niños matriculados en otras regiones distintas a la Metropolitana.

Fuente: Elaboración propia en base a la Ficha de Protección Social (FPS) e información proporcionada por Junji e Integra.

Finalmente, es plausible preguntarse si existe alguna asociación entre el grado de vulnerabilidad del hogar y la decisión de asistir al centro más cercano. Es importante notar que el grado de vulnerabilidad, medido en FPS, se calcula en función de variables tales como el número de personas capaces de generar ingresos, índices de discapacidad de los integrantes del hogar y acceso a servicios básicos (agua potable, alcantarillado y electricidad). Estas dimensiones en conjunto con las preferencias de los padres respecto de los beneficios de asistir a la enseñanza parvularia podrían incidir en que los padres envíen a sus hijos a centros más cercanos a sus hogares. La Tabla $\mathrm{N}^{\circ} 11$ compara la asistencia efectiva al establecimiento más cercano en relación a la asistencia a otros establecimientos para distintos tramos de puntajes FPS. De los resultados se observa que el porcentaje de niños que asisten al establecimiento más cercano no varía significativamente de acuerdo al puntaje FPS del hogar. Luego, 1 de cada 3 niños matriculados en Junji e Integra e inscritos en FPS, asiste al establecimiento más próximo a su hogar. 
TABLA N ${ }^{\circ}$ 11: $\quad$ COMPARACIÓN ENTRE LA ASISTENCIA EFECTIVA Y LA ASISTENCIA AL CENTRO MÁS CERCANO SEGÚN PUNTAJE EN FPS

\begin{tabular}{lcrrrr}
\hline Puntaje FPS & $\begin{array}{c}\text { Asisten al centro } \\
\text { más cercano }\end{array}$ & $\begin{array}{c}\text { Asisten a } \\
\text { otro centro }\end{array}$ & $\begin{array}{r}\text { Total } \\
\text { asistencia }\end{array}$ & $\%$ aciertos & $\begin{array}{c}\text { Total no } \\
\text { asisten }^{(b)}\end{array}$ \\
\hline $2.000-3.000$ pts. & 2.063 & 5.145 & 7.208 & $28,62 \%$ & 43.197 \\
$3.001-4.000$ pts. & 497 & 1.020 & 1.517 & $32,76 \%$ & 9.660 \\
$4.001-5.000$ pts. & 342 & 725 & 1.067 & $32,05 \%$ & 7.132 \\
5.001-6.000 pts. & 297 & 584 & 881 & $33,71 \%$ & 5.937 \\
$6.001-7.000$ pts. & 288 & 663 & 951 & $30,28 \%$ & 6.299 \\
7.001-8.000 pts. & 304 & 668 & 972 & $31,28 \%$ & 6.625 \\
más de 8.000 pts. & 904 & 1.992 & 2.896 & $31,22 \%$ & 22.927 \\
Todos & $\mathbf{4 . 6 9 5}$ & $\mathbf{1 0 . 7 9 7}$ & $\mathbf{1 5 . 4 9 2}$ & $\mathbf{3 0 , 3 1 \%}$ & $\mathbf{1 0 1 . 7 7 7}$ \\
\hline
\end{tabular}

Notas: (a) Corresponde al porcentaje de casos en que los niños asisten al centro identificado como el más cercano al hogar. (b) Agrupa a todos los niños entre 0 y 3 años que no aparecen en los registros de Junji e Integra. Sin embargo, parte de este grupo podría estar matriculado en establecimientos privados o establecimientos de enseñanza básica y media con dependencias destinadas a la enseñanza preescolar.

Fuente: Elaboración propia en base a la Ficha de Protección Social (FPS) e información proporcionada por Junji e Integra.

\section{Oferta pública de educación parvularia en la Región Metropolitana}

En esta sección analizamos la oferta de centros administrados por Junji e Integra en la Región Metropolitana a partir de dos dimensiones: i) número de centros de enseñanza preescolar por zonas geográficas y niveles educacionales y ii) capacidad (número de cupos totales y disponibles) por nivel de enseñanza.

Respecto de la distribución de los centros preescolares en la Región Metropolitana, la Tabla $\mathrm{N}^{\circ} 12$ presenta el número de establecimientos agrupados por zonas y niveles educacionales. Si bien la oferta aumenta en la medida que se incrementa la edad de los menores y el nivel de enseñanza ofrecido, este aumento no es homogéneo para todos los sectores de la región. Los sectores rurales, centro y sur registran los mayores incrementos en los niveles sala cuna menor (menores de 1 año) y medio mayor (niños de 3 años). Asimismo, para todos los niveles y sectores, existe una mayor oferta de centros Junji.

En relación a la capacidad ofrecida, la Tabla $\mathrm{N}^{\circ} 13$ muestra el número de cupos totales en centros Junji e Integra por nivel de enseñanza. De los resultados se observa que la mayoría de las salas cuna ofrecen cupos en grupos de menos de 30 niños mientras que los grupos 


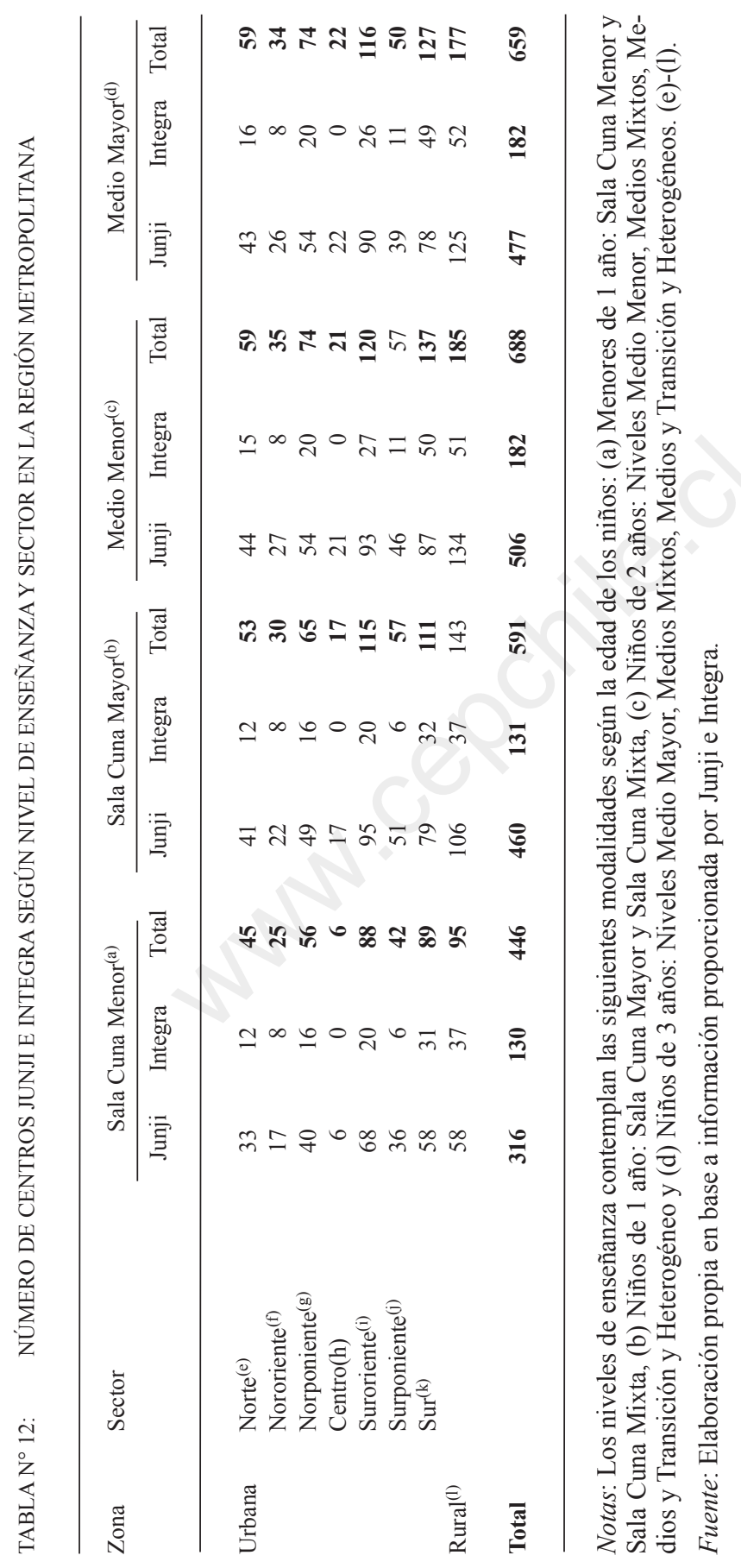




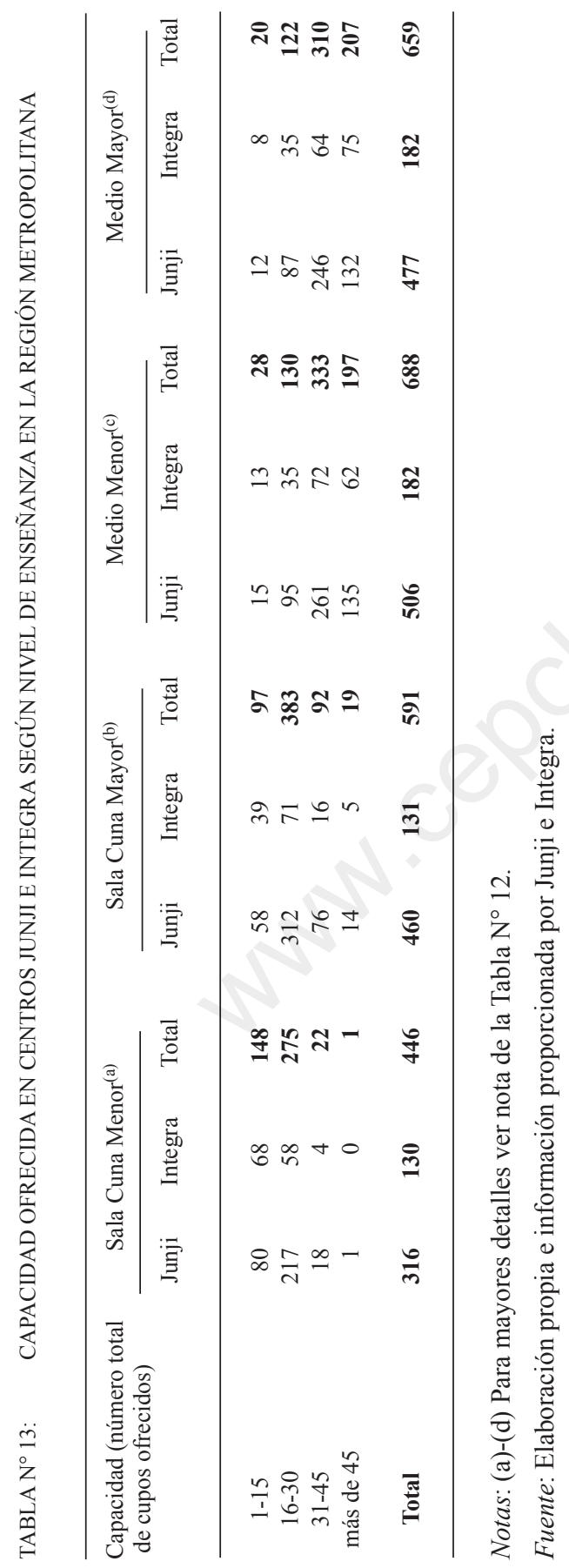


de los niveles medios están compuestos en su mayoría por más de 31 niños. A nivel de instituciones el tamaño de los grupos también difiere, de modo que la mayoría de las salas cuna Junji ofrecen cupos en grupos de entre 16 y 30 niños mientras que Integra de 1 a 15 . Para los niveles medios, los jardines Junji se componen en gran parte de 31 a 45 niños por grupo y los jardines de Integra de 16 a 30 niños por grupo. Lo anterior demuestra las diferencias entre los servicios ofrecidos por estas dos instituciones. El impacto de estas diferencias en la calidad de los servicios es un tema que está fuera del ámbito de este estudio, pero que por su importancia debe ser considerado en investigaciones futuras.

La Figura $\mathrm{N}^{\circ} 4$ presenta la distribución geográfica de los centros pertenecientes a Junji e Integra en la Región Metropolitana. El Cuadro (a) presenta el número de centros por nivel de enseñanza y el Cuadro (b) muestra la localización de la capacidad por nivel de enseñanza. Esta figura ilustra con mayor detalle los resultados de las Tablas $\mathrm{N}^{\text {os. }} 12$ y 13. En relación a la localización de la oferta (Cuadro a), el sector sur concentra la mayoría de los centros tanto en sala cuna como en nivel medio (para niños entre 0 y 1 año y 2 y 3 años de edad, respectivamente). Es interesante observar que en general el número de centros disponibles difiere de acuerdo al nivel de enseñanza, excepto en el sector nororiente. En éste se observa que existe una mínima diferencia entre el número de salas cuna y el número de centros que ofrecen servicios para niños de hasta 3 años. Sin embargo, en los sectores norponiente y sur las diferencias de disponibilidad se hacen más evidentes.

Respecto de la capacidad ofrecida por nivel de enseñanza y sector de la región, el Cuadro (b) muestra que transversal al nivel de enseñanza, la capacidad total declarada es mayor en el sector sur (lo que resulta consistente con el mayor número de centros en ese sector) en comparación al sector norte de la región.

Un aspecto interesante de analizar es el grado de concentración de los centros Junji e Integra considerando distintos rangos de distancia. Las Tablas Nos. 14 y 15 presentan el número de centros Junji cuyo establecimiento Integra más cercano se encuentra inscrito en rangos discretos de distancia. Los resultados muestran que más de la mitad de los jardines Junji tiene un centro Integra más cercano a menos de 1 kilómetro y que la distancia promedio entre ambos es aproximadamente 550 metros. 
FIGURA N ${ }^{\circ}$ 4: $\quad$ DISTRIBUCIÓN GEOGRÁFICA DE LA OFERTA DE CENTROS JUNJI E INTEGRA EN LA REGIÓN METROPOLITANA

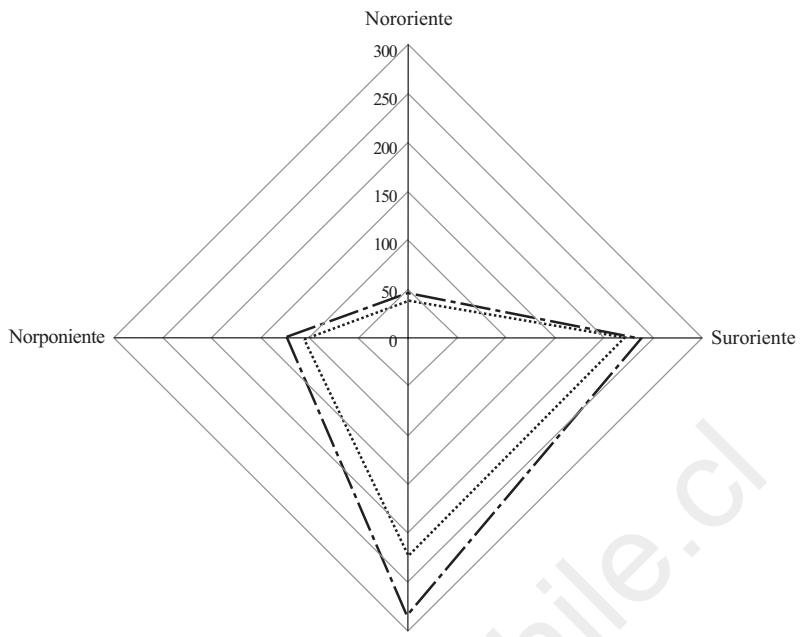

Surponiente

- - - Sala Cuna (0-1 años) _ - Nivel Medio (2-3 años)

a) Número total de centros

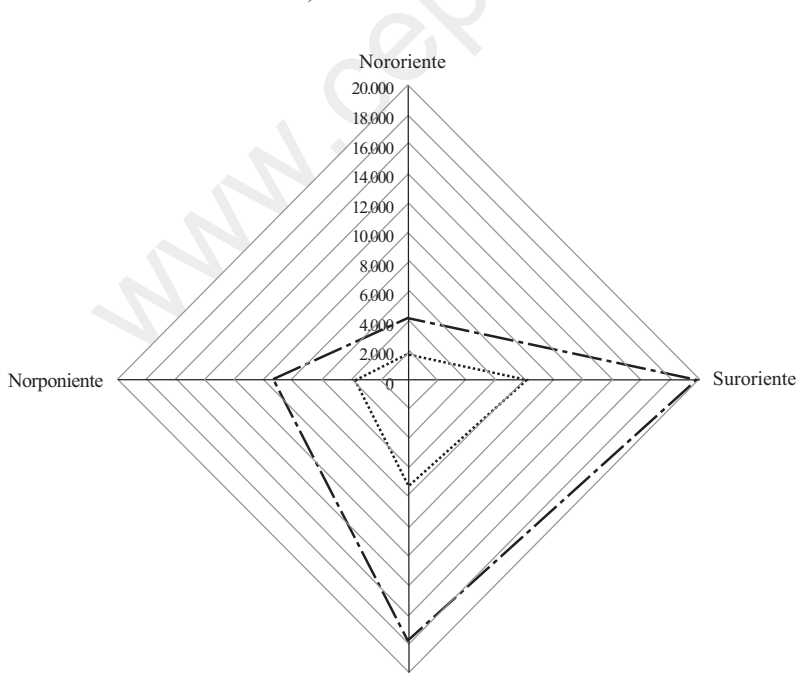

Surponiente

- - - Sala Cuna (0-1 años) _ - Nivel Medio (2-3 años)

b) Capacidad total de los centros

Notas: Para mayores detalles sobre la descripción de las comunas que componen cada sector, ver nota de la Figura $\mathrm{N}^{\circ} 2$.

Fuente: Elaboración propia en base a la Ficha de Protección Social (FPS) e información proporcionada por Junji e Integra. 
TABLA No 14: NÚMERO DE CENTROS JUNJI CUYO CENTRO INTEGRA MÁS CERCANO SE ENCUENTRA A DISTINTOS RADIOS DE DISTANCIA (Kilómetros)

\begin{tabular}{ccccc}
\hline $\begin{array}{c}\text { Distancia al } \\
\text { centro Integra } \\
\text { más cercano }\end{array}$ & $\begin{array}{c}\text { Menores } 1 \text { año } \\
\mathrm{N}^{\circ} \text { Centros } \\
\text { Junji }\end{array}$ & $\begin{array}{c}1 \text { año } \\
\mathrm{N}^{\circ} \text { Centros } \\
\text { Junji }\end{array}$ & $\begin{array}{c}2 \text { años } \\
\mathrm{N}^{\circ} \text { Centros } \\
\text { Junji }\end{array}$ & $\begin{array}{c}3 \text { años } \\
\mathrm{N}^{\circ} \text { Centros } \\
\text { Junji }\end{array}$ \\
\hline menos de $1 \mathrm{~km}$ & 143 & 195 & 250 & 243 \\
& $(52,77 \%)$ & $(48,99 \%)$ & $(58,00 \%)$ & $(60,30 \%)$ \\
$1-2 \mathrm{kms}$ & 76 & 112 & 106 & 97 \\
& $(28,04 \%)$ & $(28,14 \%)$ & $(24,59 \%)$ & $(24,07 \%)$ \\
$2-3 \mathrm{kms}$ & 27 & 29 & 43 & 26 \\
& $(9,96 \%)$ & $(10,80 \%)$ & $(6,73 \%)$ & $(6,45 \%)$ \\
$3-4 \mathrm{kms}$ & 12 & 17 & 14 & 12 \\
& $(4,43 \%)$ & $(4,27 \%)$ & $(3,25 \%)$ & $(2,98 \%)$ \\
$4-5 \mathrm{kms}$ & 5 & 8 & 5 & 5 \\
más de $5 \mathrm{kms}$ & $(1,85 \%)$ & $(2,01 \%)$ & $(1,16 \%)$ & $(1,24 \%)$ \\
& 8 & 23 & 27 & 20 \\
Total & $(2,95 \%)$ & $(5,78 \%)$ & $(6,26 \%)$ & $(4,96 \%)$ \\
& $\mathbf{2 7 1}$ & $\mathbf{3 9 8}$ & $\mathbf{4 3 1}$ & $\mathbf{4 0 3}$ \\
& $(\mathbf{1 0 0 \% )}$ & $(\mathbf{1 0 0 \% )}$ & $(\mathbf{1 0 0} \%)$ & $\mathbf{( 1 0 0 \% )}$ \\
\hline
\end{tabular}

Nota: Entre paréntesis se indica el porcentaje de centros Junji cuyo establecimiento Integra más cercano se encuentra en determinados radios de distancia como porcentaje de centros Junji en cada nivel de enseñanza.

Fuente: Elaboración propia en base a FPS e información proporcionada por Junji e Integra.

Si bien lo anterior no es necesariamente una demostración de una distribución ineficiente de los centros, sí es una señal preocupante. Esto, pues, es posible pensar en la existencia de economías de escala en la provisión de servicios de cuidado infantil. Por otra parte, la cercanía entre los centros podría generar una preocupación adicional en la medida que los establecimientos posean capacidad subutilizada. Lo anterior podría atribuirse a un mal diseño de las ubicaciones estratégicas de ambas instituciones. Análisis preliminares en esta línea indican que los centros Junji e Integra (para el segmento de niños menores a 1 año) ubicados a menos de 1 kilómetro de distancia poseen capacidades similares (es decir, los grupos que ofrecen son de tamaños similares) y que éstos se encuentran ocupados con la demanda existente ${ }^{19}$.

19 En promedio, los establecimientos analizados exceden su capacidad declarada en 1 ó 2 cupos tanto para Junji como para Integra, pero no se descarta que extendiendo el análisis a mayores radios de distancia y niveles educacionales (niños de 1 a 3 años) esta situación pudiese ser diferente. 
TABLA N ${ }^{\circ}$ 15: $\quad$ NÚMERO DE CENTROS JUNJI CUYO CENTRO INTEGRA MÁS CERCANO SE ENCUENTRA INSCRITO EN DISTINTOS RADIOS DE DISTANCIA (KILÓMETROS)

\begin{tabular}{|c|c|c|c|c|c|c|c|c|}
\hline \multirow{2}{*}{$\begin{array}{l}\text { Distancia } \\
\text { centros Integra }\end{array}$} & \multicolumn{2}{|c|}{ Menores 1 año } & \multicolumn{2}{|c|}{ Niños 1 año } & \multicolumn{2}{|c|}{ Niños 2 años } & \multicolumn{2}{|c|}{ Niños 3 años } \\
\hline & $\begin{array}{c}\mathrm{N}^{\circ} \\
\text { centros }\end{array}$ & $\begin{array}{l}\text { Distancia } \\
\text { promedio }\end{array}$ & $\begin{array}{c}\mathrm{N}^{\circ} \\
\text { centros }\end{array}$ & $\begin{array}{l}\text { Distancia } \\
\text { promedio }\end{array}$ & $\begin{array}{c}\mathrm{N}^{\circ} \\
\text { centros }\end{array}$ & $\begin{array}{l}\text { Distancia } \\
\text { promedio }\end{array}$ & $\begin{array}{c}\mathrm{N}^{\circ} \\
\text { centros }\end{array}$ & $\begin{array}{l}\text { Distancia } \\
\text { promedio }\end{array}$ \\
\hline menos de $1 \mathrm{~km}$ & 143 & 0,556 & 195 & 0,566 & 250 & 0,547 & 243 & 0,546 \\
\hline $1-2 \mathrm{kms}$ & 76 & 1,442 & 112 & 1,402 & 106 & 1,393 & 97 & 1,389 \\
\hline $2-3 \mathrm{kms}$ & 27 & 2,396 & 43 & 2,408 & 29 & 2,385 & 26 & 2,388 \\
\hline $3-4 \mathrm{kms}$ & 12 & 3,326 & 17 & 3,360 & 14 & 3,456 & 12 & 3,454 \\
\hline $4-5 \mathrm{kms}$ & 5 & 4,529 & 8 & 4,559 & 5 & 4,700 & 5 & 4,803 \\
\hline más de $5 \mathrm{kms}$ & 8 & 7,750 & 23 & 9,323 & 27 & 8,407 & 20 & 8,556 \\
\hline
\end{tabular}

Notas: Todas las distancias son expresadas en kilómetros.

Fuente: Elaboración propia en base a la Ficha de Protección Social (FPS) e información proporcionada por Junji e Integra.

\section{Capacidad, demanda potencial y matrícula efectiva en educación parvularia en la Región Metropolitana}

Hemos identificado 107.777 niños entre 0 y 3 años, inscritos en FPS y que no están matriculados en jardines Junji o Integra, pero que podrían asistir a centros de enseñanza preescolar ${ }^{20}$. Adicionalmente, hemos mostrado en términos agregados la distribución geográfica de los centros.

En esta sección, analizaremos la decisión de matrícula en centros preescolares tomando como hipótesis que ésta puede ser explicada por la disponibilidad de cupos en los establecimientos más próximos a los hogares.

La Figura $\mathrm{N}^{\circ} 5$ muestra la localización de la capacidad disponible y la demanda potencial en la Región Metropolitana. En esta figura hacemos énfasis en la capacidad de la oferta existente para responder a la demanda potencial entendida desde dos perspectivas: i) como el total de menores entre 0 y 3 años inscritos en FPS y ii) como el conjunto de menores en el mismo rango de edad en situación de vulnerabilidad socioeconómica

${ }^{20}$ Podrían estar matriculados en otro tipo de establecimientos ya sea en jardines privados o establecimientos de enseñanza básica y media con niveles preescolares. 
FIGURA N ${ }^{\circ}$ 5: $\quad$ CAPACIDAD Y DEMANDA POTENCIAL POR EDUCACIÓN PREESCOLAR EN LA REGIÓN METROPOLITANA

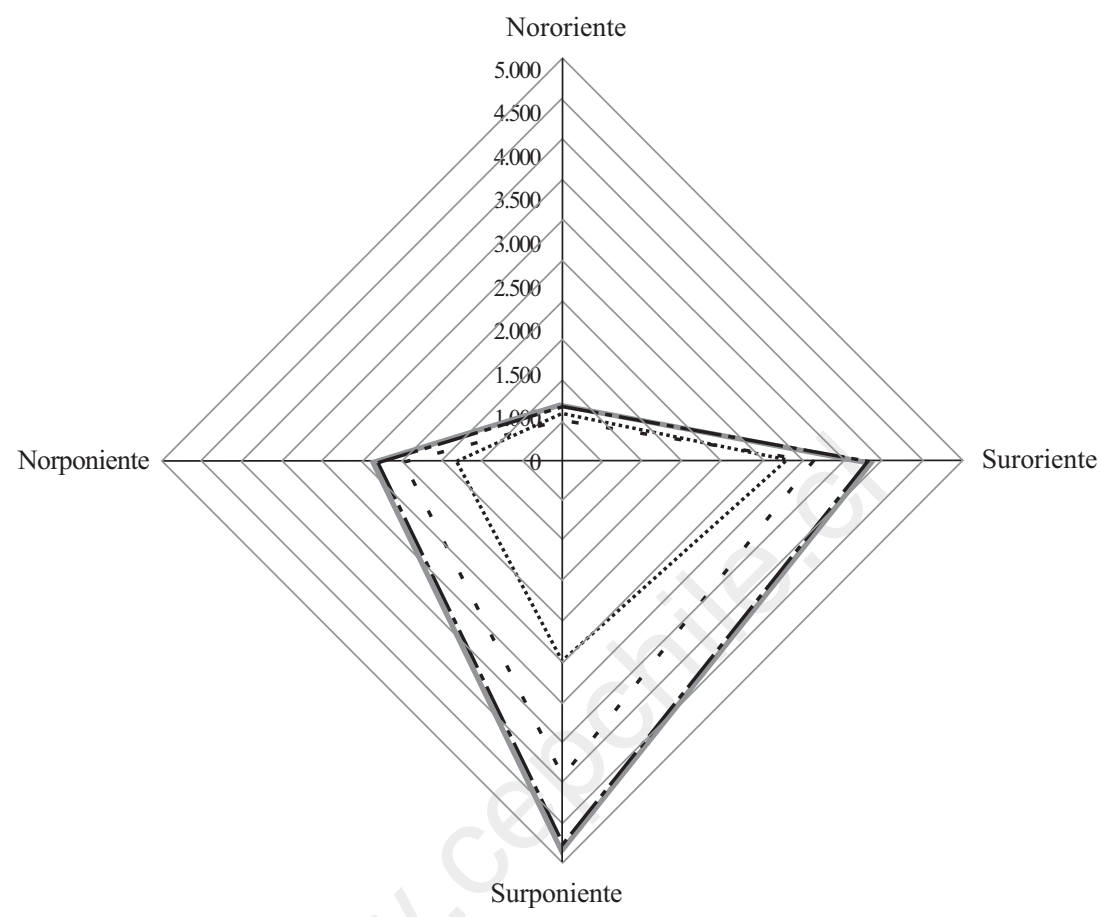

$$
\begin{aligned}
& \text {-. - Capacidad centros Junji-Integra } \\
& \text { - } \quad \text { - Niños (0-3 años) 1er. quintil FPS (<8.600 pts.) } \\
& \text { - Niños (0-3 años) en FPS } \\
& \text { — - Niños (0-3 años) 1-3er. quintil FPS (<13.483 pts.) }
\end{aligned}
$$

Notas: Para mayores detalles sobre la descripción de las comunas que componen cada sector, ver nota de la Figura $\mathrm{N}^{\circ} 2$.

Fuente: Elaboración propia en base a la Ficha de Protección Social (FPS) e información proporcionada por Junji e Integra.

definida a partir del puntaje en FPS. En este último caso, hemos considerado menores que pertenecen al primer y tercer quintil de la FPS, respectivamente, debido a que representan los grupos elegibles para recibir el beneficio de asistencia gratuita a centros Junji e Integra otorgados por el programa $\mathrm{ChCC}^{21}$.

${ }^{21}$ Para mayores detalles sobre los criterios de elegibilidad y beneficios de ChCC, véase sección 3. 
En la Figura $\mathrm{N}^{\circ} 5$ se observa que a excepción del sector nororiente, la oferta preescolar se ve ampliamente sobrepasada por la cantidad de niños entre 0 y 3 años inscritos en FPS. Si bien no todos ellos son demandantes efectivos de educación parvularia, cuando se considera el grupo de niños pertenecientes a los tres primeros quintiles de ingreso, se mantiene el exceso de demanda para todos los sectores. Es interesante notar la situación en el sector nororiente. En este sector, la capacidad (oferta) tiende a satisfacer en mayor proporción la demanda respecto de los sectores más vulnerables.

La Tabla $\mathrm{N}^{\circ} 16$ muestra la demanda potencial, capacidad total ofrecida y la matrícula declarada en centros de Junji e Integra por nivel de enseñanza y sectores de la Región Metropolitana.

En la tabla se observa que la condición de exceso de matrícula de un centro varía según el nivel educacional y sector de la Región Metropolitana, de modo que, en promedio, las salas cuna tienen excedida su capacidad en un $6,61 \%$ (20.646 cupos versus 22.011 matriculados), mientras que los niveles medios presentan una holgura de capacidad equivalente al 4,87\% de su capacidad (50.795 cupos versus 48.434 matriculados).

Considerando los anteriores resultados, la Tabla $\mathrm{N}^{\circ} 17$ muestra el número de establecimientos cuya capacidad declarada excede, es igual o superior a la matrícula para cada nivel y sector de la Región Metropolitana. Encontramos entonces que para los niveles de sala cuna menor y sala cuna mayor, la gran mayoría de los centros tiene su capacidad excedida por los actuales niños matriculados, mientras que en los niveles medios se observa lo contrario.

A partir del análisis anterior podemos concluir que en el eventual escenario de un aumento de demanda efectiva por parte de las familias más vulnerables, el sistema no tendría la capacidad (al menos en el corto plazo) de poder asegurar servicios de cuidado infantil para un número significativo de hogares. En este contexto, comprender en detalle los factores que determinan la demanda por estos servicios es un elemento de suma importancia para efectos de las políticas públicas. A continuación desarrollamos estimaciones conducentes a responder esta interrogante. 


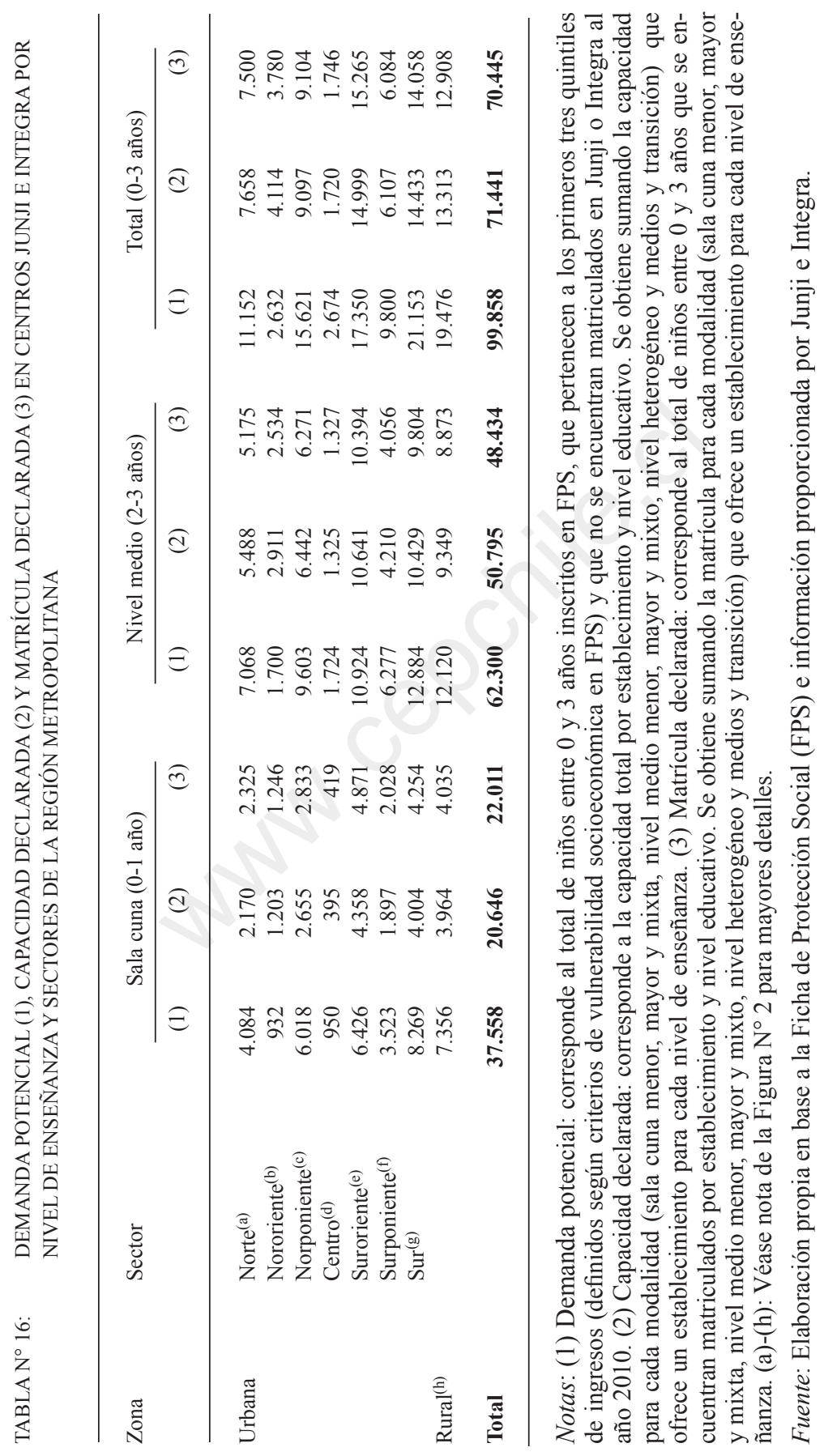




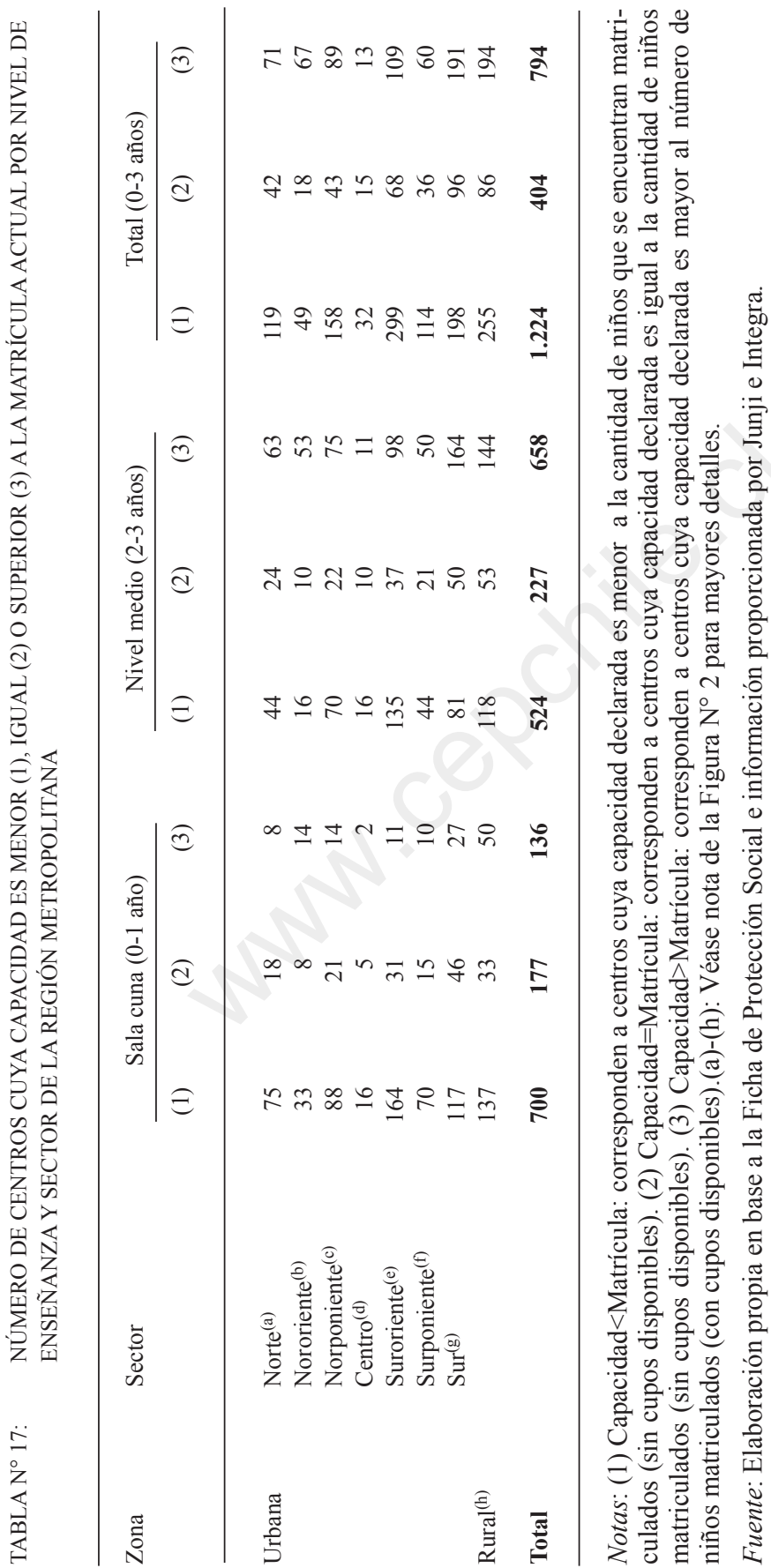




\subsection{Modelo de decisión para la asistencia a la educación preescolar}

Utilizando un modelo de elección discreta e información de FPS estimamos la probabilidad de que el menor asista a un centro preescolar (niños hasta 3 años de edad). En nuestro análisis, la variable dependiente es la matrícula del menor en Junji o Integra al año 2010 y controlamos por la edad, género, nivel educacional, situación ocupacional de los padres y la distancia desde el hogar hasta el jardín más cercano asignado de acuerdo al nivel educacional al que le corresponde asistir al menor según su edad. La Tabla $\mathrm{N}^{\circ} 18$ presenta la estadística descriptiva de la muestra utilizada.

La Tabla $\mathrm{N}^{\circ} 19$ presenta los resultados para tres especificaciones distintas del modelo. Los resultados del modelo base (primera columna) de la Tabla $\mathrm{N}^{\circ} 19$, sugieren que la probabilidad de que un menor esté matriculado en un centro Junji o Integra está positivamente afectada por la edad del menor (un año adicional incrementa la probabilidad de asistir al jardín en 6,2 puntos porcentuales) y negativamente afectada por el nivel educacional de los padres. En particular, los resultados de la primera columna indican que los hijos de madres con más de doce años de escolaridad tienen 2 puntos porcentuales menos de probabilidad de asistir a un centro Junji o Integra en relación a los hijos de madres con menos de ocho años de escolaridad. Del mismo modo, hijos con padres con más de doce años de escolaridad tienen 2,4 puntos porcentuales menos de probabilidad de asistir a un centro Junji o Integra en relación a los hijos de padres con menos de ocho años de escolaridad.

Controlando por la situación ocupacional de los padres (columna 2 de la Tabla $\mathrm{N}^{\circ}$ 19), los resultados anteriores se mantienen. Esto es, niños de mayor edad tienen mayor probabilidad de asistir a centros Junji e Integra (equivalente a 6,2 puntos porcentuales adicionales en probabilidad de asistencia) y menor probabilidad de asistencia en la medida que sus padres posean mayores niveles de escolaridad (aproximadamente 2,4 y 2,7 puntos porcentuales extras en la probabilidad para hijos cuyas madres y padres tienen estudios superiores, respectivamente). Es interesante considerar el efecto de la condición de empleabilidad de los padres sobre la asistencia a educación preescolar. Nuestros resultados indican que, en relación a las madres inactivas, los hijos de madres ocupadas y desocupadas tienen entre 2,2 y 3,5 puntos porcentuales extras en la probabilidad de asistir a salas cuna y jardines Junji e Integra. Si bien puede parecer contraintuitivo que los hijos de madres desemplea- 


\begin{tabular}{|c|c|c|c|c|c|}
\hline Variable & Obs. & Promedio & Desv. Est. & Min. & $\operatorname{Max}$ \\
\hline Edad & 116.976 & 1,907 & 0,947 & 0 & 3 \\
\hline Género & 116.976 & 0,515 & 0,499 & 0 & 1 \\
\hline Madre ausente & 116.976 & 0,245 & 0,430 & 0 & 1 \\
\hline Padre ausente & 116.976 & 0,592 & 0,491 & 0 & 1 \\
\hline Madre con menos de 8 años de escolaridad & 116.976 & 0,079 & 0,269 & 0 & 1 \\
\hline Madre con 8-11 años de escolaridad & 116.976 & 0,277 & 0,447 & 0 & 1 \\
\hline Madre con 12 años de escolaridad & 116.976 & 0,342 & 0,474 & 0 & 1 \\
\hline Madre con más de 12 años de escolaridad & 116.976 & 0,055 & 0,229 & 0 & 1 \\
\hline Padre con menos de 8 años de escolaridad & 116.976 & 0,047 & 0,213 & 0 & 1 \\
\hline Padre con 8-11 años de escolaridad & 116.976 & 0,154 & 0,361 & 0 & 1 \\
\hline Padre con 12 años de escolaridad & 116.976 & 0,171 & 0,377 & 0 & 1 \\
\hline Padre con más de 12 años de escolaridad & 116.976 & 0,033 & 0,179 & 0 & 1 \\
\hline Madre ocupada & 116.976 & 0,330 & 0,470 & 0 & 1 \\
\hline Madre desocupada & 116.976 & 0,025 & 0,157 & 0 & 1 \\
\hline Madre inactiva & 116.976 & 0,398 & 0,489 & 0 & 1 \\
\hline Padre ocupado & 116.976 & 0,377 & 0,484 & 0 & 1 \\
\hline Padre desocupado & 116.976 & 0,020 & 0,141 & 0 & 1 \\
\hline Padre inactivo & 116.976 & 0,009 & 0,098 & 0 & 1 \\
\hline $\begin{array}{l}\text { Jardín más cercano ubicado a menos de } 1 \mathrm{~km} \\
\text { del hogar }\end{array}$ & 116.976 & 0,886 & 0,316 & 0 & 1 \\
\hline $\begin{array}{l}\text { Jardín más cercano ubicado entre } 1 \text { y } 2 \text { kms } \\
\text { del hogar }\end{array}$ & 116.976 & 0,084 & 0,277 & 0 & 1 \\
\hline $\begin{array}{l}\text { Jardín más cercano ubicado entre } 2 \text { y } 3 \mathrm{kms} \\
\text { del hogar }\end{array}$ & 116.976 & 0,013 & 0,114 & 0 & 1 \\
\hline $\begin{array}{l}\text { Jardín más cercano ubicado entre } 3 \text { y } 4 \text { kms } \\
\text { del hogar }\end{array}$ & 116.976 & 0,003 & 0,061 & 0 & 1 \\
\hline Jardín más cercano ubicado a más de $4 \mathrm{kms}$ & & & & & \\
\hline $\begin{array}{l}\text { del hogar } \\
\text { Quintil 1 FPS }\end{array}$ & $\begin{array}{l}116.976 \\
116.976\end{array}$ & $\begin{array}{l}0,012 \\
0,810\end{array}$ & $\begin{array}{l}0,109 \\
0,392\end{array}$ & $\begin{array}{l}0 \\
0\end{array}$ & $\begin{array}{l}1 \\
1\end{array}$ \\
\hline Quintil 2 FPS & 116.976 & 0,136 & 0,343 & 0 & 1 \\
\hline Quintil 3 FPS & 116.976 & 0,035 & 0,184 & 0 & 1 \\
\hline Quintil 4 FPS & 116.976 & 0,013 & 0,115 & 0 & 1 \\
\hline Quintil 5 FPS & 116.976 & 0,004 & 0,066 & 0 & 1 \\
\hline
\end{tabular}

Notas: Género toma el valor 1 para hombres y 0 para mujeres. Las variables de ausencia de la madre y del padre toman el valor 1 si el menor vive con otro familiar (abuelos o tíos) distinto a los padres o padrastros. Respecto del nivel educacional de los padres, la categoría base corresponde a madres/padres con menos de 8 años de escolaridad. Si la madre o padre no vive con el menor, las variables de nivel educacional toman el valor 0. En relación al estado ocupacional de los padres, la categoría base corresponde a madres/padres inactivos. Por último, la categoría base de distancias hogar-centro más cercano corresponde a jardines situados a 1 o menos kilómetros del hogar del menor. En este caso, los centros más cercanos al hogar son asignados de acuerdo al nivel educacional al que el menor le corresponde asistir en relación a su edad. Todas las especificaciones incluyen efectos fijos por comuna de residencia.

Fuente: Elaboración propia en base a la Ficha de Protección Social e información proporcionada por Junji e Integra. 
TABLA N ${ }^{\circ} 19$ : EFECTO MARGINAL SOBRE LA PROBABILIDAD DE ASISTIR A UN
CENTRO PARA NIÑOS ENTRE 0 Y 3 AÑOS INSCRITOS EN FPS QUE
VIVEN EN LA REGIÓN METROPOLITANA

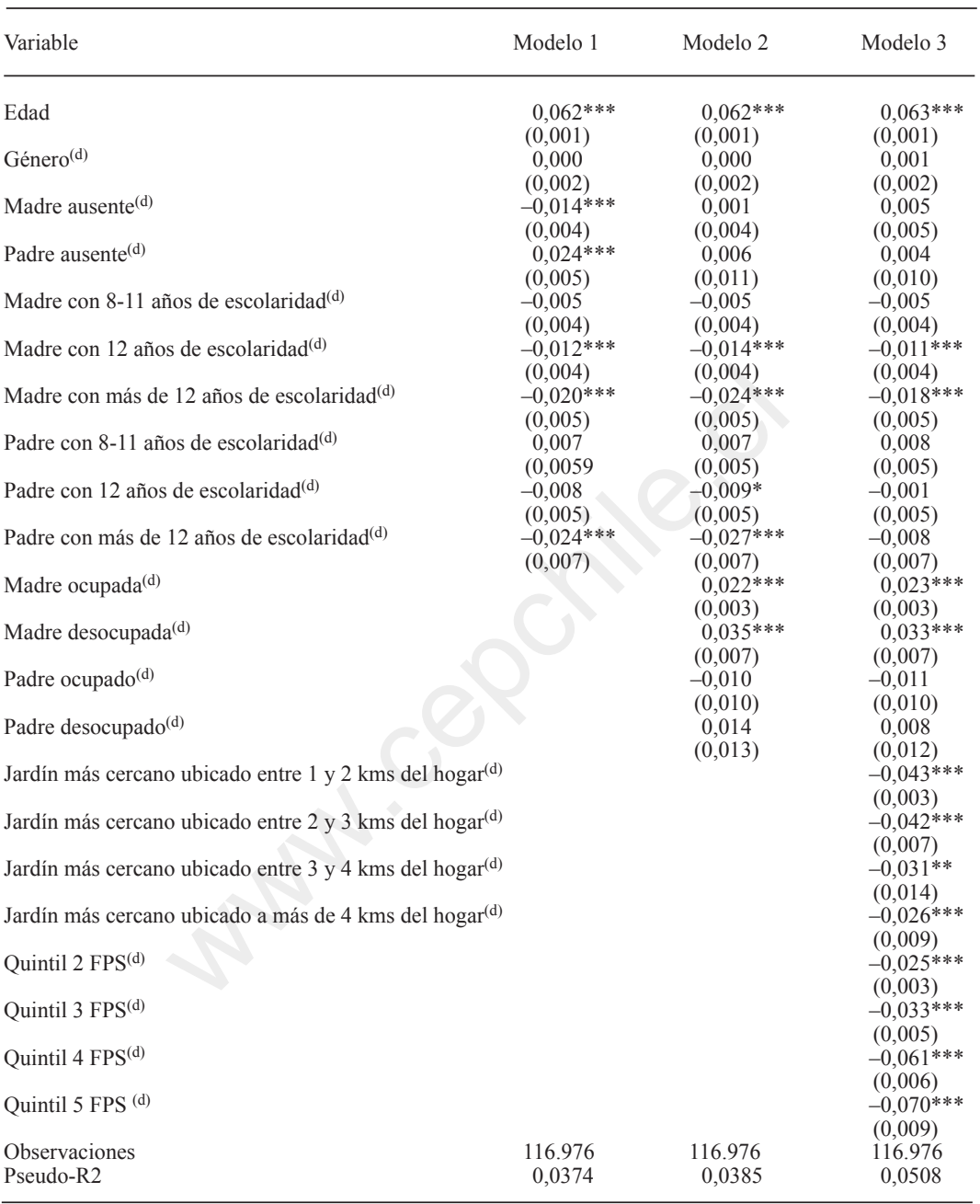

Notas: Género toma el valor 1 para hombres y 0 para mujeres. Las variables de ausencia de la madre y del padre toman el valor 1 si el menor vive con otro familiar (abuelos o tíos) distinto a los padres o padrastros. Respecto del nivel educacional de los padres, la categoría base corresponde a madres/padres con menos de 8 años de escolaridad. Si la madre o padre no vive con el menor, las variables de nivel educacional toman el valor 0 . En relación al estado ocupacional de los padres, la categoría base corresponde a madres/padres inactivos. Por último, la categoría base de distancias hogar-centro más cercano corresponde a jardines situados a 1 o menos kilómetros del hogar del menor. En este caso, los centros más cercanos al hogar son asignados de acuerdo al nivel educacional al que el menor le corresponde asistir en relación a su edad. Todas las especificaciones incluyen efectos fijos por comuna de residencia. (d) Indica que la estimación del efecto marginal se realizó sobre una variable dummy.

Fuente: Elaboración propia en base a la Ficha de Protección Social e información proporcionada por Junji e Integra. 
das tengan más probabilidad de asistir a jardines infantiles que los hijos de madres ocupadas, los resultados pueden explicarse porque dichas madres manifiestan una intención de encontrar empleo y realizan los esfuerzos para conseguirlo, lo cual involucra tiempo y la necesidad de dejar a sus hijos en centros preescolares. Por otra parte, madres empleadas también pueden hacer uso de los beneficios de sala cuna o jardín infantil que por ley están obligados a ofrecer las empresas de más de veinte trabajadoras, lo que hace menos probable la matrícula en un centro público. Por su parte, la condición de empleabilidad de los padres pareciera tener un efecto poco claro y no significativo sobre la probabilidad de asistencia a centros de Junji e Integra.

Las estimaciones que incorporan la distancia entre el hogar y el centro más cercano (asignado de acuerdo al nivel educacional correspondiente a la edad del menor) muestran que ésta tiene un efecto negativo sobre la probabilidad de asistencia (columna 3 de la Tabla $\left.\mathrm{N}^{\circ} 19\right)$ ). De este modo, en la medida que se incrementa la distancia entre el hogar y los centros, la probabilidad de asistir a Junji o Integra disminuye entre 4,3 y 2,6 puntos porcentuales (relativo a vivir a un kilómetro o menos del establecimiento más cercano). Adicionalmente, los resultados indican que en la medida que disminuye la vulnerabilidad socioeconómica del hogar de los menores (medida a través del quintil del hogar en FPS), disminuye la probabilidad de asistir a salas cuna y jardines Junji e Integra. Específicamente, aquellos niños cuyos hogares pertenecen al segundo quintil de FPS tienen una menor probabilidad de asistir a centros preescolares de estas instituciones (2,5 puntos porcentuales menos), mientras que aquellos del quinto quintil tienen menos probabilidad ( 7 puntos porcentuales menos) de asistir a dichos establecimientos que los niños que pertenecen al primer quintil de FPS. Estos resultados demuestran la importancia de la ubicación geográfica y las condiciones socio-económicas de los hogares en la decisión de enviar un menor a centros preescolares públicos.

\section{Conclusiones}

Este trabajo constituye el primer análisis de demanda y oferta de la educación preescolar en Chile. En él, hemos utilizado información de niños entre 0 y 3 años (y sus hogares) inscritos en la Ficha de Protección Social (FPS) y registros de matriculados en Junji e Integra al año 
2010, para caracterizar a la población que asiste a centros preescolares, a quienes potencialmente podrían hacerlo y a los proveedores de educación preescolar. Nuestro enfoque otorga especial énfasis a la ubicación de la demanda (potencial y efectiva) y la oferta (capacidad). En particular, utilizamos la distancia hogar-centro para evaluar si la ubicación de los centros preescolares responde a la existencia de una demanda potencial y efectiva cercana, si existen decisiones estratégicas detrás de la localización de los centros pertenecientes a distintos proveedores y si la inasistencia está influenciada por la oferta de cupos disponibles en los centros cercanos al hogar.

Los resultados indican que la distancia hogar-centro es un elemento importante en la decisión de asistencia a salas cuna y jardines infantiles. Dentro del segmento de niños matriculados, la distancia efectiva hogar-centros es 2 kilómetros mientras que la distancia promedio al centro más cercano al hogar es 720 metros. Esto sugiere que si bien los padres no escogen el centro más cercano para enviar a sus hijos, escogen un centro ubicado en un radio cercano al hogar (probablemente circunscrito a la comuna de residencia). En el caso de los menores no matriculados, la distancia promedio desde el hogar al jardín más cercano es 820 metros.

Nuestros resultados demuestran que la probabilidad de asistir a un centro preescolar se ve afectada positivamente por la edad de los menores y la condición de empleabilidad de las madres. Por otra parte, la probabilidad de asistencia disminuye con la educación de la madre y vulnerabilidad del hogar. La evidencia también indica que la distancia hogar-centro más cercano influye en la probabilidad de asistir a la enseñanza preescolar. Después de controlar por la educación y el estado ocupacional de los padres, el género y edad de los menores, se observa que, en promedio, vivir a dos o más kilómetros del jardín más cercano al hogar disminuye la probabilidad de asistencia a centros Junji e Integra en 3,3 puntos porcentuales respecto de vivir a una menor distancia del centro más cercano al hogar.

Nuestro análisis también entrega indicios respecto de problemas en la distribución de la oferta. Encontramos sectores donde la oferta es baja (especialmente en los niveles de salas cuna). Además, identificamos centros tanto con exceso de matrícula como con holguras de capacidad. Finalmente, encontramos gran cercanía entre centros Junji e Integra. 
En este trabajo también analizamos la institucionalidad que rige la educación preescolar. Identificamos cuatro proveedores de educación parvularia de los cuales tres son los más representativos. Si bien Junji e Integra apuntan al mismo segmento de niños y ofrecen programas y modalidades educativas similares, están poco coordinados al momento de decidir la ubicación de los centros y parecen actuar de manera autónoma.

Finalmente, consideramos importante destacar que existe además un importante segmento privado del cual poco se conoce, especialmente por el carácter voluntario de la certificación (y supervisión) de centros privados que realiza Junji. Lo anterior ha dificultado la existencia de un directorio actualizado de todas las instituciones y centros que proveen enseñanza preescolar y desde ahí la regulación y fiscalización en el sector. Éste es un tema de gran importancia que debiera ser explorado.

\section{REFERENCIAS BIBLIOGRÁFICAS}

Barnet, S. (1995). "Long Term Effects of Early Childhood Programs on Congnitive and School". The Future of Children, Vol. 5, ํ 3; 25-50.

Behrman J. y S. Urzúa (2012). "Economic Perspectives on Some Important Dimensions of Early Childhood Development in Developing Countries". En P. R. Britton, P. L. Engle, y C. M. Super (eds.), Handbook of Early Child Development: Translating Research to Global Policy. Oxford University Press.

Bernal R. y M. P. Keane (2010). "Quasi-Structural Estimation of a Model of Child Care Choices and Child Cognitive Ability Production". Journal of Econometrics, Vol. 156, Nㅜ 1; 164-189.

(2011). "Child Care Choices and Children's Cognitive Achievement:

The Case of Single Mothers". Por aparecer en Journal of Labor Economics.

Carneiro P. y J. J. Heckman (2003). "Human Capital Policy”. En IZA Disussion Paper $\mathrm{N}^{\circ} 821$.

Chumacero R., D. Gomez y R. Paredes (2008). "I Would Walk 500 Miles (if it Paid)”. Working Paper, MPRA 15125.

Congreso Nacional de Chile (2009). Ley 20.379 que crea el Sistema Intersectorial de Protección Social e Institucionaliza el Subsistema de Protección Integral a la Infancia Chile Crece Contigo.

Cuhna, F., J. J. Heckman y L. Lochner (2005). "Interpreting the Evidence on Life Cycle Skill Formation". Handbook of the Economics of Education, Vol. 1; 697-812.

Elacqua G., M. Schneider y J. Buckley (2006). "School Choice in Chile: Is it Class or Classroom". Journal of Policy Analysis and Management, Vol. $25, \mathrm{~N}^{\circ} 3,577-601$. 
Engle P. L., M. M. Black, J. R. Behrman, M. Cabral de Mello, P. J. Gertler, L. Kapiriri, R. Martorell, M. Eming Young (2007). "International Child Development Steering Committee. Strategies to Avoid the Loss of Potential among 240 Million Children in the Developing World". The Lancet $369 ; 229-242$.

Gallego, F. y A. Hernando (2008). "On the Determinants and Implications of School Choice: Semi-Structural Simulations for Chile”. Documento de Trabajo, Instituto de Economía, Pontificia Universidad Católica de Chile, $\mathrm{N}^{\circ} 343$.

Gallego F. y A. Hernando (2009). "School Choice in Chile: Looking at the Demand Side". Documento de Trabajo, Instituto de Economía, Pontificia Universidad Católica de Chile, №356.

Heckman, J. J. (2006). "Skill Formation and the Economics of Investing in Disadvantaged Children". Science, Vol. 312; 1900-1902.

Herbst, C. y E. Tekin (2010). "Distance Matters: Proximity to Social Service Agencies and the Utilization of Child are Subsidies". National Bureau of Economic Research, $\mathrm{N}^{\circ} 16250$.

Kitano, S. y J. Uda (2007). "Analysis of the Provision of the Early Childhood Services: A comparative and Experimental Study of an Under-populated and a Urbana Area in Japan". International Journal of Child Care and Education Policy, Vol. 1, $\mathrm{N}^{\circ}$ 1; 73-84.

Knudsen, E. I., J. J. Heckman, J. L. Cameron y J. P. Shonkoff (2006). "Economic, Neurobiological, and Behavioral Perspectives on Building America's Future Workforce". Proceeding of the National Academy of Sciences of the Unites States of America, PNAS, Vol. 103, N ${ }^{\circ} 27$; 10155-10162.

Ministerio de Educación (Mineduc) (2001). "La Educación Parvularia en Chile”. Serie Educación Parvularia 2001: Aportes a la Reflexión y a la Acción, Vol. 1.7.

(2009). "Caracterización de los Hogares e Identificación de las Razones por las cuales No Matriculan a sus Hijos e Hijas de 4 años en el Primer Nivel de Transición a la Escuela". Estudio desarrollado por el Observatorio Social de la Universidad Alberto Hurtado y ProUrbana de la Pontificia Universidad Católica de Chile para el Ministerio de Educación.

Ministerio de Planificación (Mideplan) (2010). Cuatro años creciendo juntos: Memoria de la Instalación del Sistema de Protección Integral a la Primera Infancia: Chile Crece Contigo 2006-2010.

Noboa-Hidalgo, G. y S. Urzúa (2012). "The Effects of Participation in Public Childcare Centers: Evidence from Chile”. Journal of Human Capital, Vol. $6 \mathrm{~N}^{\circ} 1$ (Spring 2012).

Sapelli, C. y A. Torche (2002). "Subsidios al Alumno o a la Escuela: Efectos sobre la Elección de Colegios Públicos". Cuadernos de Economía., Año $39, \mathrm{~N}^{\circ} 117 ; 175-202$.

Schady, N. (2005). "Early Childhood Development in Latin America and the Caribbean”. World Bank Policy Research, Working Paper N 3869. 
Shonkoff, J. P. y D. Phillips (2000). "From Neurons to Neighborhoods The Science of Early Childhood Development". National Research Council, Institute of Medicine.

Sinnott, R. W. (1984). "Virtues of the Haversine". Sky and Telescope 98 (2), 159.

Tokman, A. (2010). "Radiografía de la Educación Parvularia Chilena: Desafíos y Propuestas". Serie de Políticas Públicas UDP, Documentos de Trabajo, $\mathrm{N}^{\circ} 5$. 